\title{
3 Research Square

\section{Simulation and Control of High-Order Flatness in Rolling Wide Titanium Strip With 20-High Mill}

\section{Guanyu Zhou}

University of Science and Technology Beijing

Hui Li

University of Science and Technology Beijing

Anrui He ( $\nabla$ harui_ustb@163.com )

University of Science and Technology Beijing

Chao Liu

University of Science and Technology Beijing

\section{Wenquan Sun}

University of Science and Technology Beijing

\section{Simeng Chen}

University of Science and Technology Beijing

Zhengqiao Liu

Hunan Xiangtou Goldsky Titanium Metal Co.,Ltd

Cong Han

Hunan Xiangtou Goldsky Titanium Metal Co.,Ltd

\section{Original Article}

Keywords: 20-high mill, Wide titanium strip, High-order flatness defect, Adjustment characteristics, Finite element method

Posted Date: January 29th, 2021

DOI: https://doi.org/10.21203/rs.3.rs-155420/v1

License: (c) (1) This work is licensed under a Creative Commons Attribution 4.0 International License.

Read Full License 


\title{
Simulation and control of high-order flatness in rolling wide titanium strip with 20-high mill
}

\author{
Guanyu Zhou ${ }^{1}$, Hui Li ${ }^{1}$, Anrui He ${ }^{1 *}$, Chao Liu ${ }^{1}$, Wenquan $\mathrm{Sun}^{2}$, Simeng Chen ${ }^{2}$, Zhengqiao Liu ${ }^{3}$, Cong Han ${ }^{3}$ \\ ${ }^{1}$ National Engineering Research Center for Advanced Rolling Technology, University of Science and Technology Beijing, Beijing \\ 100083, China \\ ${ }^{2}$ National Engineering Technology Research Center of Flat Rolling Equipment, University of Science and Technology Beijing, Beijing \\ 100083, China \\ ${ }^{3}$ Hunan Xiangtou Goldsky Titanium Metal Co. , Ltd, Changsha 410006, China
}

\begin{abstract}
In wide titanium strip cold rolling process, the high-order flatness defect is one of the most difficult problem to be solved. Based on finite element method, considering the anisotropic mechanical characteristics of titanium, an implicit integration calculation model of rolls-strip for 20-high mill was developed, which can simulate the dynamic rolling process. The model was used to analyze the adjustment characteristics of high-order flatness on the 20-high mill. The simulation revealed as the increasing of the $1 \# \& 7$ \# AS-U or $2 \# \& 6$ \# AS-U press adjustment, the high-order flatness defect was more aggravated; And as the increasing of $3 \#$ \& 5\# AS-U or 4\# AS-U press adjustment, the high-order flatness defect was alleviated to some extent. In addition, the high-order flatness cannot be effectively adjusted by roll shifting. Finally, the industrial test showed that increasing $4 \#$ AS-U press adjustment can effectively relieve the high-order flatness defect.
\end{abstract}

Keywords: 20-high mill; Wide titanium strip; High-order flatness defect; Adjustment characteristics; Finite element method

\section{Introduction}

Commercially pure titanium strip is a widely used titanium metal material, and its mechanical properties anisotropy and microstructure evolution law have attracted the attention of numerous scholars. Liu et al. [1] studied the anisotropy and forming characteristics of commercially pure titanium sheet under different rolling paths and found that cross rolling showed a smaller planar anisotropy. Liu et al. [2] studied the effects of cold rolling and annealing on the microstructure evolution of commercially pure titanium sheet and found that twinning was the dominant deformation mechanism when the cold rolling reduction is less then $40 \%$, that $20 \%$ cold rolled sheet recrystallized almost completely when the annealing time reached $60 \mathrm{~min}$. Roth et.al. [3] studied the anisotropy of mechanical behavior of commercially pure $\alpha$-Ti samples cut along the rolling and transverse direction under tensile tests and suggested a mechanism based on the consideration of the dislocation glide anisotropy.

Compared to the research on the microstructure of commercially pure titanium, the research on the shape control of titanium strips in cold rolling process is not sufficient. Zhu et.al [4] analyzed the ridge buckle defect of the pure titanium cold rolling strip in the production process. Shi et.al [5] studied the distribution of tensile stress of titanium strip in a continuous annealing furnace with FEM method.

The simulation model of a 20-high mill has been researched for a long time by a large number of scholars. Wang et.al [6] used the influence function method to establish the elastic deformation model of the 20-high rolling mill. Yu et.al [7] proposed a new 20-high mill method that can mesh element depended on the contact length between rolls or between the work roll and the strip. Zhang et,al [8] introduced the method of the finite element stiffness matrix assembly when using the influence function method to calculate the roll deformation and coupled the plastic flatness model based on the threedimensional difference method to establish a 20-high mill roll system and strip integrated rapid calculation model. Yuan et.al [9] proposed that the roll was regarded as a semi-infinite body with finite length, and based on the boundary integral equation method, an accurate roll-to-roll

* Corresponding author: Anrui He E-mail: harui@ustb.edu.cn 
flattening model was established. In addition, the 20-high mill simulation model based on the finite element method has high calculation accuracy because of few assumptions, but it is suitable for offline calculation due to the lengthy calculation time. Zhang et.al [10] established a threedimensional 20-high mill finite element model with ANSYS and studied the contact pressure between rolls and the shape of the load roll gap. Li et.al [11] used the finite element method to establish a 20-high mill roll system and strip integrated elastic-plastic deformation statics model.

Besides the research on the simulation model of a 20high mill, some scholars have also studied the quarter waves control of the 20-high mill. Hara et.al.[12] found that when rolling stainless steel on a 20-high mill, quarter waves could be effectively corrected by giving a concave profile partially to the 1 st intermediate taper rolls. Faced with the disadvantages of difficulty in processing the concave roll contour of the 1 st intermediate taper rolls, Kim et.al [13] studied the control ability of the intermediate multi-section taper roll contour on the quarter waves and found that quarter waves could be reduced remarkably by changing taper mode of the 1 st intermediate roll. Aizawa et.al [14] found that the shape control range of quarter waves side and edge waves side was doubled by the introduced of FSBAs.

Currently, when cold rolling wide titanium strip with $1250 \mathrm{~mm}$ as the main width on a 20-high mill, it exhibits more complex flatness defect. During the cold rolling process from the entry thickness of $4.0 \mathrm{~mm}$ to the thickness of the finished product of $0.4 \mathrm{~mm}$, the shape meter near the area $100 \mathrm{~mm}-300 \mathrm{~mm}$ from the edge shows compressive stress which indicating that this region and adjacent regions have uneven extension in the rolling direction, and the compression stress region extends relatively long, as shown in Fig.1. When rolling to thin gauges below $1.6 \mathrm{~mm}$, high-order waves begin to appear in the compressive stress area, as shown in Fig.2. Compared with quarter waves, it is closer to the edge. This research calls it high-order flatness defect.

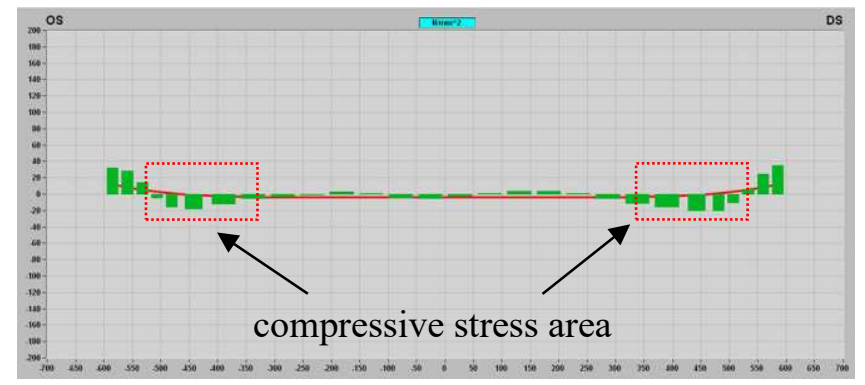

Figure 1. Stress distribution on the shape meter.
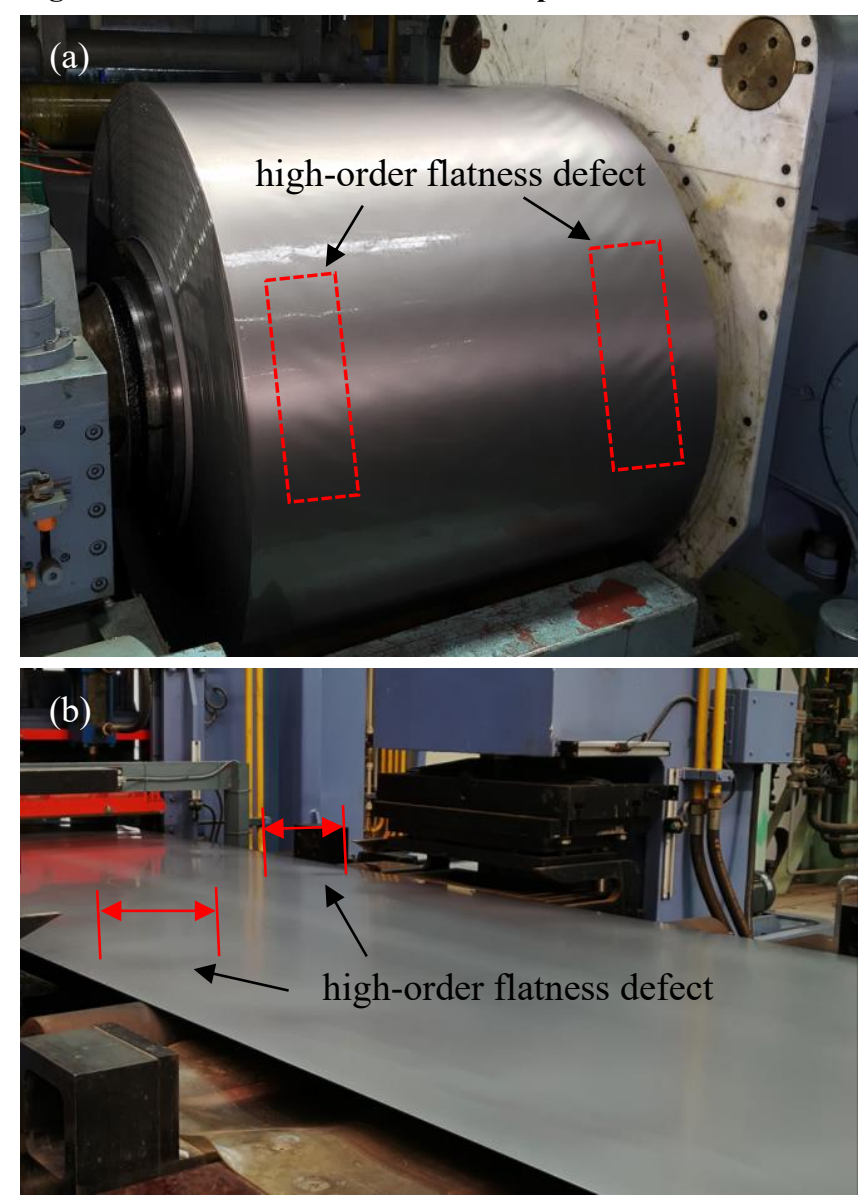

Figure 2. High-order flatness defect. (a) during coiling; (b) during straightening.

The complex high-order flatness defect mentioned above proposes a new research subject, and it is necessary to study the high-order flatness defect control characteristics of 20-high mill. The roll system of the 20high rolling mill has a complex structure, and the small roll diameter work roll is more prone to high-order deflection under the action of the rolling force. Coupled with the characteristics of anisotropic material in the elastoplastic stage of commercially pure titanium, the deformation behavior of the roll system and strip becomes quite complicated. Therefore, the study of high-order flatness defect requires a higher-precision calculation model. 
However, the rolls-strip coupling model established by many scholars for 20-high mill with FEM belongs to the static model, which cannot realize the dynamic rolling between work rolls and strip. As a result, the contact arc length under the static model is twice than the actual situation, as shown in Figure 3. Thus, this research uses ABAQUS finite element software to establish an implicit integration calculation model of rolls-strip for 20-high mill that can realize dynamic rolling between work rolls and strip and analyzes the adjustment characteristics of shape control means.
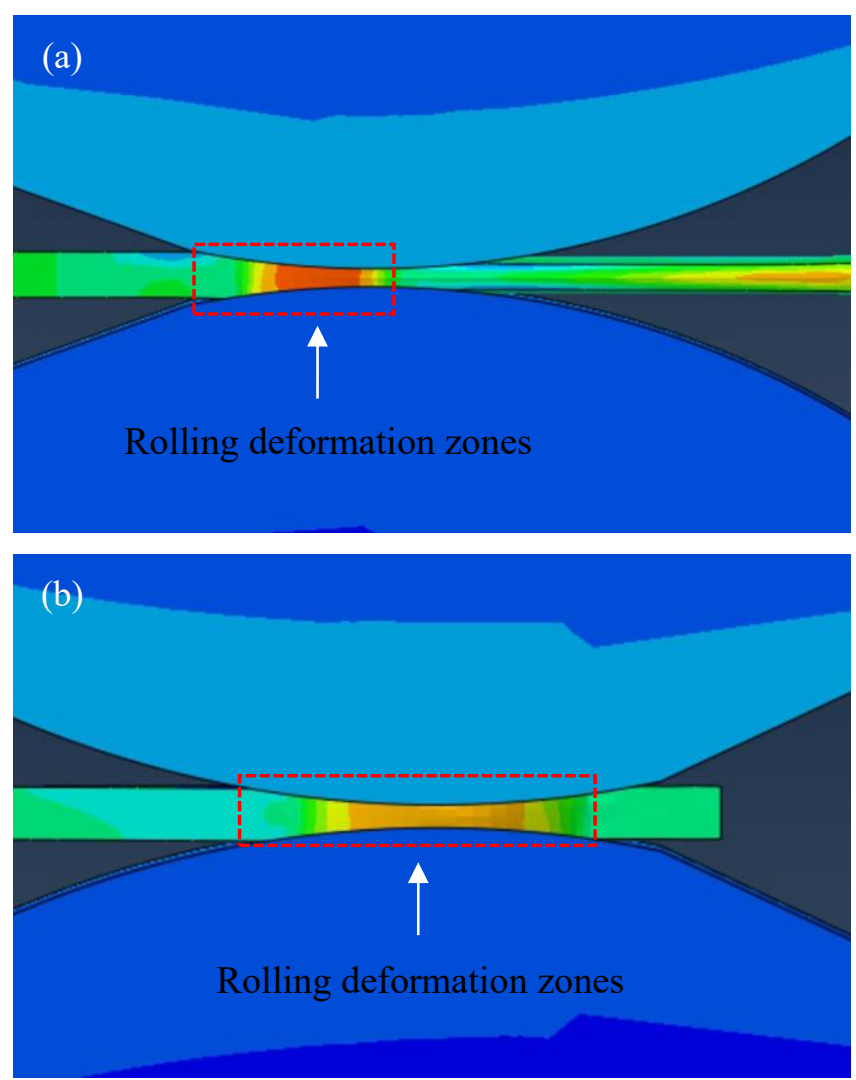

Figure 3. Rolling deformation area. (a) under rotation model; (b) under static model.

\section{Implicit FEM of rolls-strip integrated for 20-high mill}

\subsection{The structure of 20-high mill roll system}

The roll system of 20-high mill is shown in Figure 4. The backing bearing is A, B, C, D, E, F, G, H, the 2nd intermediate roller is $\mathrm{I}, \mathrm{J}, \mathrm{K}, \mathrm{L}, \mathrm{M}, \mathrm{N}$, the 1 st intermediate roller is $\mathrm{O}, \mathrm{P}, \mathrm{Q}, \mathrm{R}$, and the work roller is $\mathrm{S}, \mathrm{T}$. The 20 -high mill is equipped with two kinds of shape control modes: crown adjustment of back-up rolls (AS-U) and the 1st intermediate taper roll online shifting. The overlapping length of the taper surface of the 1 st intermediate roll and the titanium strip is the amount of roll shifting. The rack of the AS-U adjustment mechanism is located between the B and $\mathrm{C}$ backup rolls. The AS- $\mathrm{U}$ adjustment mechanism had seven segments eccentric rings that could realize independent adjustment, as shown in Figure 5. Relative to the zero position of eccentric ring, the seven segments can realize eccentricity in the range of $-0.28 \mathrm{~mm}$ to $+0.28 \mathrm{~mm}$, and the percentage can be adjusted from $-100 \%$ to $+100 \%$.

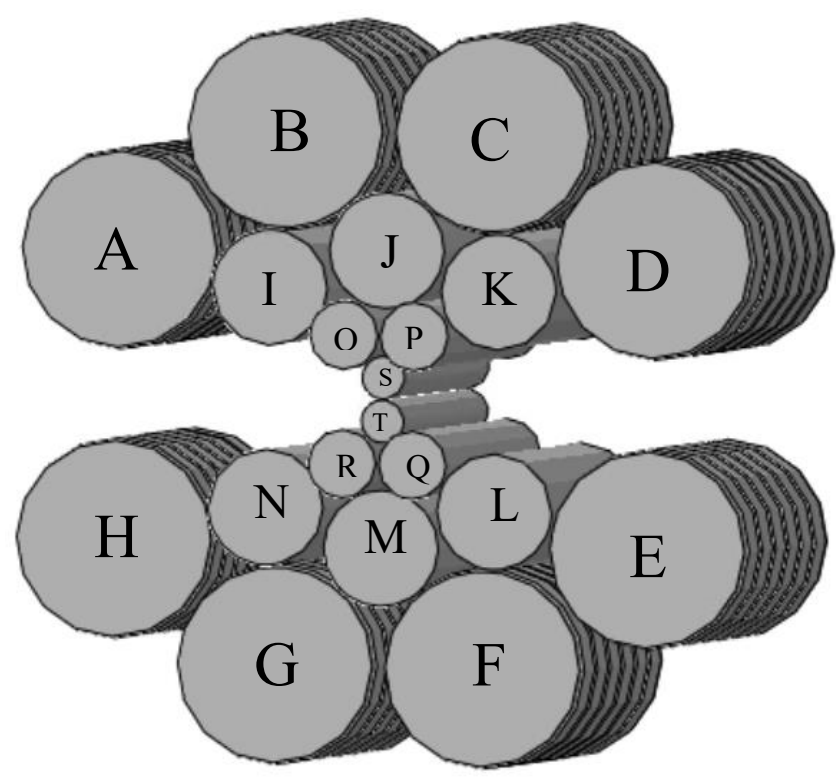

Figure 4. Roll system structure of 20-high mill.

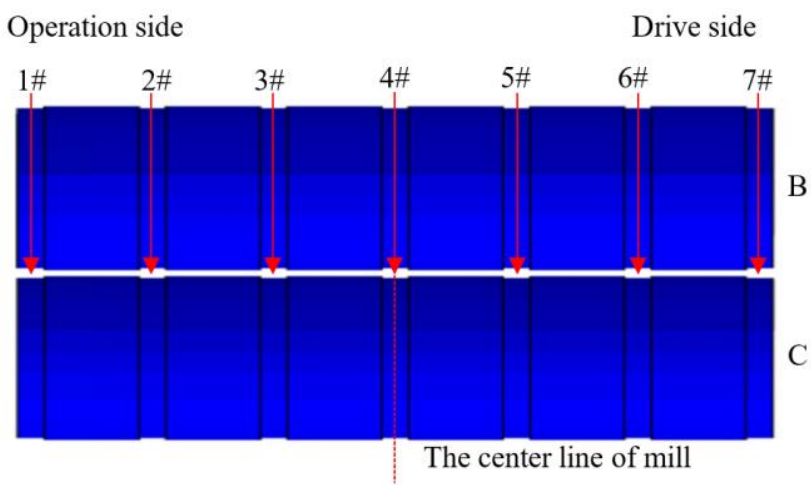

Figure 5. The AS-U adjustment means of 20-high mill.

\subsection{The model geometric parameters}

The geometric dimensions of the rolls of 20-high mill are given in Table 1. The backup roll is comprised of mandrel, eccentric ring, backing bearing, and saddle. Ignoring the influence of the gap between these components, the backup roll is simplified into an integrated stepped structure, as shown in Figure 6. The 2nd intermediate roll and work roll adopt flat roll contour. The 1 st intermediate roll adopts $230 \mathrm{~mm} \times 0.1 \%$ single-sided taper roll contour (radius roll contour). The length of the 
titanium strip is $40 \mathrm{~mm}$ and the width is $1250 \mathrm{~mm}$, the thickness is $1.54 \mathrm{~mm}$.

Table 1. Geometrical dimensions of rolls.

\begin{tabular}{ccc}
\hline Roll & $\begin{array}{c}\text { Length } \\
/ \mathrm{mm}\end{array}$ & $\begin{array}{c}\text { Diameter } \\
/ \mathrm{mm}\end{array}$ \\
\hline Backup roll & Fig.6 & Fig.6 \\
Second middle driving roll & 1384 & 173 \\
Second middle idler roll & 1444 & 173 \\
Intermediate roll & 1580 & 102 \\
Work roll & 1414 & 63.5 \\
\hline
\end{tabular}

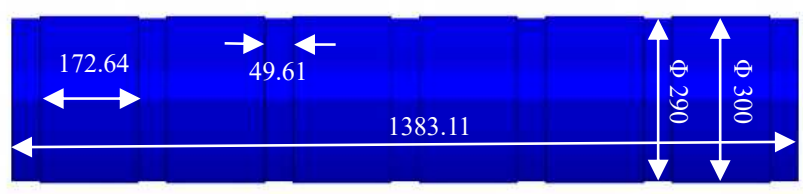

Figure 6. The simplified structure of backup roll.

\subsection{The model material parameters}

In order to calculate the deflection and flattening state of the roll more accurately, the roll is defined as an elastic material in this model, as shown in Table 2.

Table 2. Roll material parameters.

\begin{tabular}{ccc}
\hline Roll & $\begin{array}{c}\text { Elasticity } \\
/ \mathrm{MPa}\end{array}$ & $\begin{array}{c}\text { Poisson } \\
\text { ratio }\end{array}$ \\
\hline Backup roll & $2.19 \times 105$ & 0.3 \\
Second middle roll & $2.05 \times 105$ & 0.265 \\
Intermediate roll & $2.05 \times 105$ & 0.265 \\
Work roll & $2.18 \times 105$ & 0.28 \\
\hline
\end{tabular}

The strip material is commercially pure titanium TA2, the main component (mass fraction $\%, \mathrm{Fe} \leq 0.3, \mathrm{C} \leq 0.1$, $\mathrm{N} \leq 0.05, \mathrm{H} \leq 0.015, \mathrm{O} \leq 0.25$, the rest is Ti). Because the symmetry of the hexagonal close-packed structure of pure titanium is not as good as that of body-centered cubic and face-centered cubic, the slip system and twinning modes are different when loading in different directions, so that under the same conditions, the anisotropy of the closepacked hexagonal structure is stronger. In order to make the characteristics of mechanical anisotropy of the strip be considered in the model, the strip is defined as an elastoplastic material with orthotropic anisotropy. Li et.al [15] studied the relationship between engineering constants and elastic matrix with the hexagonal crystal system structure. Wang et.al [16] proposed that the elastic matrix of the hexagonal crystal system has 5 independent components. The elastic matrix of commercially pure titanium hexagonal crystal system expressed by engineering constants can be given:

$$
\left[\begin{array}{cccccc}
\frac{1}{E_{1}} & -\frac{\mu_{12}}{E_{1}} & -\frac{\mu_{13}}{E_{1}} & 0 & 0 & 0 \\
-\frac{\mu_{12}}{E_{1}} & \frac{1}{E_{2}} & -\frac{\mu_{23}}{E_{2}} & 0 & 0 & 0 \\
-\frac{\mu_{13}}{E_{1}} & -\frac{\mu_{23}}{E_{2}} & \frac{1}{E_{2}} & 0 & 0 & 0 \\
0 & 0 & 0 & \frac{1}{G_{12}} & 0 & 0 \\
0 & 0 & 0 & 0 & \frac{2\left(1+\mu_{23}\right)}{E_{2}} & 0 \\
0 & 0 & 0 & 0 & 0 & \frac{1}{G_{12}}
\end{array}\right]
$$

where 1 is the rolling direction, 2 is the lateral direction, and 3 is the thickness direction, 1, 2, 3 are the orthotropic principal axes, the rolling direction is the direction of the symmetrical principal axis of the hexagonal crystal system. $E_{i}$ is the elastic modulus in the $i$ direction, $G_{i j}$ is the shear modulus between $i$ and $j$ direction, $\mu_{i j}$ is the Poisson's ratio of the shrinkage (extension) in the $j$ direction caused by the tensile (compressive) stress in the $i$ direction.

There are 5 independent elastic coefficients of commercially pure titanium TA2, which are $E_{1}, E_{2}, \mu_{12}, \mu_{23}$, $G_{12}$, the elastic stage parameters of commercially pure titanium TA2 can be obtained through the room temperature tensile test, as shown in Table 3.

Table 3. Material parameters of commercially pure titanium TA2 in elastic stage.

\begin{tabular}{ccc}
\hline Variable description & Parameters & Value \\
\hline \multirow{2}{*}{ Elastic modulus /MPa } & $E_{1}$ & 102000 \\
& $E_{2}$ & 112000 \\
Poisson's ratio & $\mu_{12}$ & 0.33 \\
Shear modulus /MPa & $\mu_{23}$ & 0.29 \\
\hline
\end{tabular}

The deformation resistance of commercially pure titanium TA2 in the rolling direction is obtained through the compression test at room temperature, as shown in Table 4.

Table 4. Cold rolling deformation resistance of commercial pure titanium TA2.

\begin{tabular}{cc}
\hline Ture strain & Yield stress $/ \mathrm{MPa}$ \\
\hline 0 & 439 \\
0.1 & 596 \\
0.2 & 695 \\
0.3 & 766 \\
\hline
\end{tabular}




\section{4}

\section{3}

In the research on plastic deformation of commercially pure titanium with significant anisotropy, Toussaint et.al [17] used the Hill'48 anisotropic yield criterion to simulate the stamping process of pure titanium scoliosis. Xue et.al [18] considered the material anisotropy when studying the deep drawing of commercially pure titanium and adopted the Hill' 48 anisotropic yield criterion in the finite element simulation. In this study, the Hill'48 anisotropic yield criterion is used to describe the plastic deformation process of titanium strip. The Hill' 48 anisotropic yield criterion is expressed by the following equation:

$$
\begin{aligned}
& F\left(\sigma_{22}-\sigma_{33}\right)^{2}+G\left(\sigma_{33}-\sigma_{11}\right)^{2}+H\left(\sigma_{11}-\sigma_{22}\right)^{2} \\
& +2 L{\sigma_{23}}^{2}+2 M{\sigma_{31}}^{2}+2 N{\sigma_{12}}^{2}=1
\end{aligned}
$$

where $F, G, H, L, M, N$ is the material parameters characterizing the anisotropy.

The material parameters characterizing the anisotropy can be calculated:

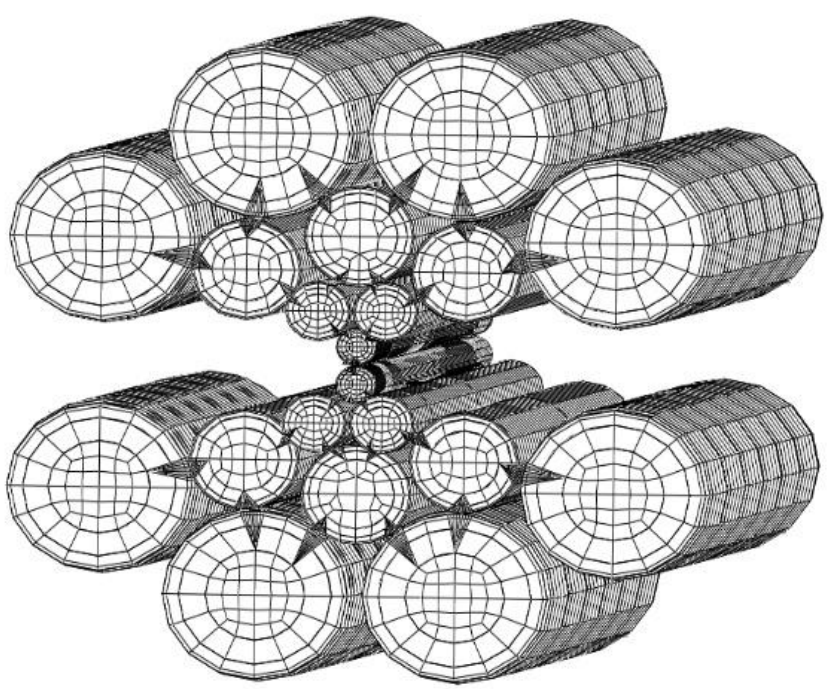

$\left\{\begin{array}{l}F=\frac{1}{2 \sigma_{0}^{2}}\left(\frac{1}{R_{22}^{2}}+\frac{1}{R_{33}^{2}}-\frac{1}{R_{11}^{2}}\right), L=\frac{3}{2 \sigma_{0}^{2} R_{23}^{2}} \\ G=\frac{1}{2 \sigma_{0}^{2}}\left(\frac{1}{R_{22}^{2}}+\frac{1}{R_{33}^{2}}-\frac{1}{R_{11}^{2}}\right), M=\frac{3}{2 \sigma_{0}^{2} R_{13}^{2}} \\ H=\frac{1}{2 \sigma_{0}^{2}}\left(\frac{1}{R_{22}^{2}}+\frac{1}{R_{33}^{2}}-\frac{1}{R_{11}^{2}}\right), N=\frac{3}{2 \sigma_{0}^{2} R_{12}^{2}}\end{array}\right.$

where $\sigma_{0}$ is the reference yield stress, the yield stress in the rolling direction is used as the reference yield stress. $R_{11}, R_{22}, R_{33}$ is the anisotropic yield stress ratio of principal axis $1,2,3, R_{12}, R_{13}, R_{23}$ is the anisotropic shear yield stress ratio relative to the principal axis $3,2,1$.

The anisotropic yield stress ratio can be calculated:

$$
\left\{\begin{array}{l}
R_{11}=\frac{\sigma_{X}}{\sigma_{0}}, R_{12}=\sqrt{3} \frac{\tau_{T}}{\sigma_{0}} \\
R_{22}=\frac{\sigma_{Y}}{\sigma_{0}}, R_{23}=\sqrt{3} \frac{\tau_{R}}{\sigma_{0}} \\
R_{33}=\frac{\sigma_{Z}}{\sigma_{0}}, R_{13}=\sqrt{3} \frac{\tau_{S}}{\sigma_{0}}
\end{array}\right.
$$

where $\sigma_{X}, \sigma_{Y}, \sigma_{Z}$ is the yield stress in anisotropic principal axis direction, $\tau_{T}, \tau_{R}, \tau_{S}$ is the shear yield stress relative to the anisotropic principal axis. Because the metal sheet is thin, $\tau_{R}$ and $\tau_{S}$ cannot be obtained, this study

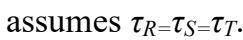

Wang et.al [19] studied the anisotropic yield stress of commercially pure titanium TA2. This study determined the anisotropic yield stress ratio based on its research results, as shown in Table 5.

Table 5. Commercial pure titanium TA2 anisotropic yield stress ratio.

\begin{tabular}{cc}
\hline Parameters & Value \\
\hline$R_{11}$ & 1.00 \\
$R_{22}$ & 1.27 \\
$R_{33}$ & 1.32 \\
$R_{12}$ & 0.99 \\
$R_{13}$ & 0.99 \\
\hline
\end{tabular}

\subsection{Mesh}

In order to ensure the calculation accuracy of the model, the mesh size is refined in the contact area between the rolls and the contact area between the rolls and strip. Considering that the grid torsion is too large in the process of rolling large deformation, and the linear reduction integral element solves the displacement more accurately when the grid is deformed by torsion. Therefore, the roll and the strip adopt the eight-node linear hexahedral noncoordinating element (C3D8R), as shown in Figure 7.

Figure. 7 The FEM model of 20-high mill with the mesh refinement near the contact area.

\subsection{Boundary conditions and loading settings}

The model realizes the contact between rolls, work rolls and the titanium strip by defining contact pairs. There are 26 contact pairs in the model. The model will not consider the friction between the rolls. According to field production experience and the friction coefficient curve of the Stone cold rolling [20], the friction coefficient between the work roll and the titanium strip is set 0.05 . 
The tie restraint method is adopted in the model to fix the rigid ring at the position of the eccentric ring corresponding to the backup roll, and the backup roll pressing and AS-U functions are realized by applying constraints and movement to the reference point of the rigid ring. At the same time, the reference point is set at the center of the end faces of the work roll. The rotation of the work roll is realized by applying constraints and movement to the reference point.

\subsection{Model evaluation}

To ensure the accuracy of numerical experiments, this study verifies the section profile and rolling force. The section profile of the titanium strip after rolling is measured online using an ultrasonic thickness gauge with a resolution of $1 \mu \mathrm{m}$. The industry verification conditions of the FEM model are as shown in Table 6. The average value of the thickness deviation between the measured profile and the simulated profile is $0.65 \%$, as shown in Figure 8 . When the thickness of the simulated exit center point is in agreement with the measured thickness of the exit center point, the deviation of the rolling force between the measured rolling force and the simulated rolling force is $8.18 \%$. Both the profile and the rolling force deviation are within $10 \%$, indicating that the model has high calculation accuracy.

Table 6. The industry verification conditions of the FEM model.

\begin{tabular}{cc}
\hline Parameters & Value \\
\hline Width strip/mm & 1250 \\
Entrance thickness/mm & 1.555 \\
Exit thickness/mm & 1.208 \\
Entrance tension/MPa & 70 \\
Exit tension/MPa & 130 \\
Amount of roll shift/mm & 63 \\
& $-36 \% 、 19 \% 、 69 \%$ 、 \\
1\# AS-U to 7\# AS-U & $12 \% 、 54 \% 、-5 \%$ - \\
adjustment amount & $61 \%$ \\
\hline
\end{tabular}

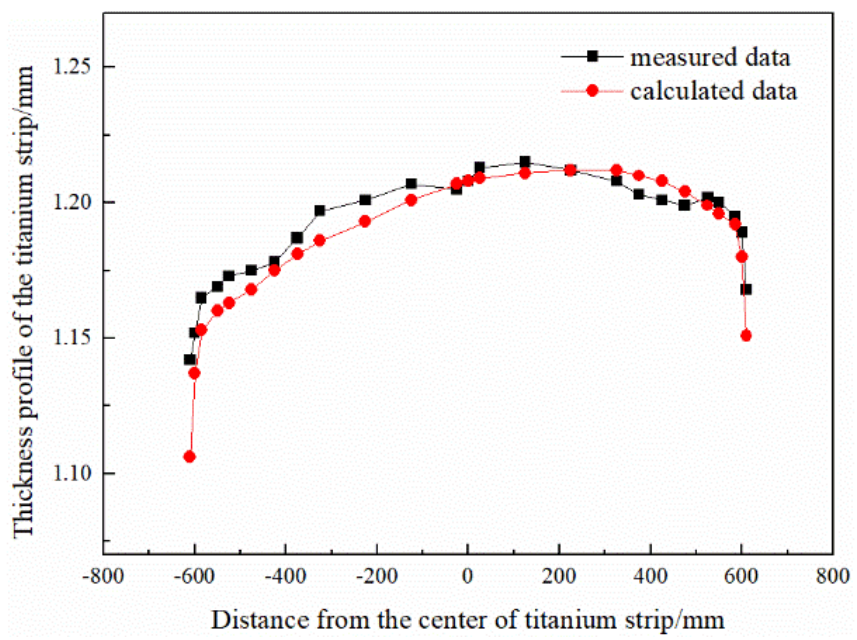

Figure. 8 The comparison of measured and simulated thickness profiles.

\section{Results and discussion}

\subsection{Lateral thickness difference after rolling without shape adjustment}

In this part, the FEM model established above is used to calculate the rolled lateral thickness difference of the titanium strip without the shape adjustment. The simulation conditions are as shown in Table 7.

Table 7. The simulation conditions without shape adjustment.

\begin{tabular}{cc}
\hline Parameters & Value \\
\hline Width strip/mm & 1250 \\
Entrance thickness/mm & 1.540 \\
Exit thickness/mm & 1.211 \\
Entrance tension/MPa & 70 \\
Exit tension/MPa & 130 \\
Amount of roll shift $/ \mathrm{mm}$ & 0 \\
1\# AS-U to 7\# AS-U & $0 \%$ \\
adjustment amount &
\end{tabular}

The lateral thickness difference after rolling is given in Figure 9. There is a local depression near the $100 \mathrm{~mm}-300$ $\mathrm{mm}$ from the edge. This study defines the $100 \mathrm{~mm}-300 \mathrm{~mm}$ from the edge as the high-order flatness defect area, which is consistent with the compressive stress of the shape meter. It shows that the occurrence of high-order flatness defect is related to the local thickness reduction of the strip after rolling. 


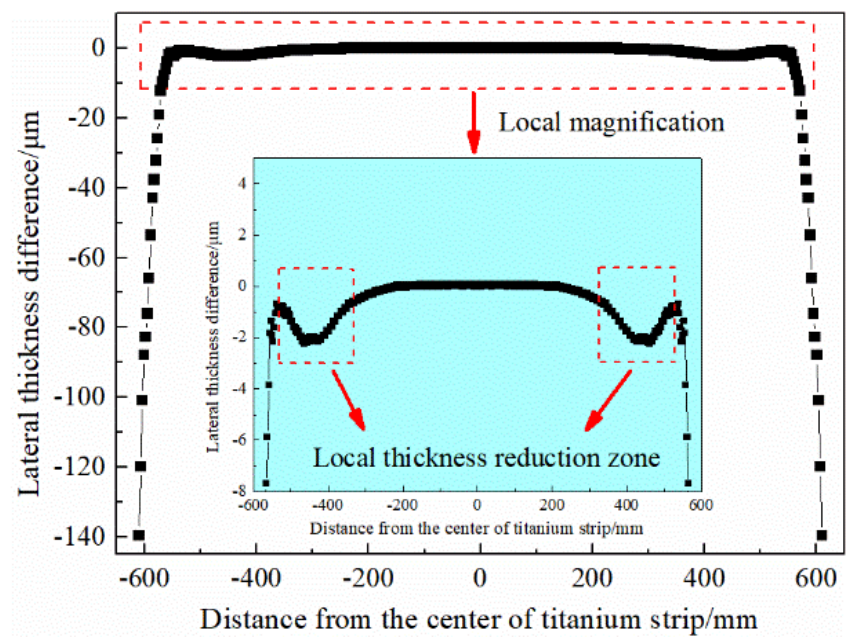

Figure. 9 Lateral thickness difference after rolling without shape adjustment.

In order to quantify the degree of depression in the highorder flatness defect area, according to the characteristics of depression in the thickness profile after rolling, the value of depression in the high-order flatness defect area can be calculated:

$$
\gamma=\frac{\left(h_{e}+h_{c}\right)}{2}-h_{d}
$$

where $\gamma$ is the value of depression in the high-order flatness defect area. $h_{e}$ is the thickness of the highest point near the edge of the titanium strip in the high-order flatness defect area, in order to reduce the influence of the volatility error of the simulation data, generally take the average value in the range of $10 \mathrm{~mm}$ near the highest point of the titanium strip edge. $h_{c}$ is the thickness of the middle part of the titanium strip, in order to reduce the influence of the volatility error of the simulation data, generally take the average thickness in the range of $200 \mathrm{~mm}$ from the center of the titanium strip. $h_{d}$ is the thickness of the lowest point in the high-order flatness defect area, in order to reduce the influence of the volatility error of the simulation data, generally take the average thickness in the range of $10 \mathrm{~mm}$ near the lowest thickness point.

The cold rolling process is a high tension rolling process, and the width of the strip is small. When the lateral thickness of the strip has uneven changes, it will cause uneven distribution of the longitudinal extension of the strip along the transverse direction, resulting flatness defect. Zhao et.al [21] studied the influence of lateral metal flow on the residual stress during hot rolling, and gave the relationship between the thickness and length of the strip before and after rolling without considering the lateral flow.
If the strip is divided into several equal width fiber strips along the transverse direction, the relationship between the length and thickness of a certain position fiber strip before and after rolling can be calculated:

$$
l(y)=\frac{H(y)}{h(y)} \times L(y)
$$

where $H(y)$ is the thickness before rolling at the $y$ position. $L(y)$ is the length before rolling at the y position. $h(y)$ is the thickness after rolling at the $y$ position. $l(y)$ is the length after rolling at the $y$ position.

The lowest thickness after rolling in the high-order flatness defect area can be calculated:

$$
h_{\gamma}=h_{\text {aim }}-\gamma
$$

where $h_{\gamma}$ is the lowest thickness after rolling in the highorder flatness defect area. $h_{\text {aim }}$ is the target thickness after rolling in the high-order flatness defect area, this study is $1.211 \mathrm{~mm} . \gamma$ is calculated by formula (5).

The rolled length of the target thickness and the lowest thickness in the high-order flatness defect area can be calculated:

$$
\left\{\begin{array}{c}
l_{\text {aim }}=\frac{\boldsymbol{H}}{\boldsymbol{h}_{\text {aim }}} \times \boldsymbol{L} \\
l_{\gamma}=\frac{\boldsymbol{H}}{\boldsymbol{h}_{\gamma}} \times \boldsymbol{L}
\end{array}\right.
$$

where $H$ is the thickness before rolling in the high-order flatness defect area, this study is $1.540 \mathrm{~mm}$. $L$ is the length before rolling in the high-order flatness defect area, this study is $1000 \mathrm{~mm}$. $h_{\text {aim }}$ and $h_{\gamma}$ can be calculated by formula (7).

Flatness expressed by relative length difference can be calculated:

$$
\lambda=\frac{\Delta l}{l} \times 10^{5}
$$

where $\lambda$ is flatness value. $\Delta l$ is the difference between the free fiber length after rolling and the standard fiber length after rolling. $l$ is the standard fiber length after rolling.

By substituting formula (8) into equation (9):

$$
\lambda_{\gamma}=\frac{h_{\text {aim }}-h_{\gamma}}{h_{\gamma}} \times 10^{5}
$$

where $\lambda_{y}$ is the flatness value caused by the local depression in the high-order flatness defect area, unit is IU.

The value of depression in the high-order flatness defect area $(\gamma)$ is $1.7 \mu \mathrm{m}$, and the flatness value caused by the local 
depression in the high-order flatness defect area $\left(\lambda_{y}\right)$ is 140.6 IU. This shows that the local depression in the thickness profile after rolling will increase the length extension in the high-order flatness defect area, which leads to the high-order flatness defect.

\subsection{Lateral thickness difference after rolling under}

\section{AS-U press adjustment}

The AS-U press adjustment is one of the essential shape control methods. It can adjust the lateral thickness of the titanium strip in real time through seven segments of eccentric rings. The diversity of transverse thickness control can be achieved through multiple combinations of 7 segments eccentric rings. In order to ensure the symmetry of the thickness profile in rolling process to prevent the occurrence of strip centerline deviation, in real time adjustment, the symmetrical position AS-U adjustment change should be as consistent as possible. Therefore, the AS-U press adjustment that is symmetrically distributed along the centerline of 20-high mill is basic form of AS-U adjustment. It is easy to know that the symmetrical position AS-U lifting adjustment characteristics should be opposite to the press adjustment characteristics. This study analyzes the adjustment characteristics of the symmetrical position AS-U press adjustment method to the high-order flatness defect. The simulation conditions are as shown in Table 8 .

Table 8. The simulation conditions under AS-U press adjustment.

\begin{tabular}{cc}
\hline Parameters & Value \\
\hline Width strip/mm & 1250 \\
Entrance thickness/mm & 1.540 \\
Exit thickness/mm & 1.211 \\
Entrance tension/MPa & 70 \\
Exit tension/MPa & 130 \\
Amount of roll shift $/ \mathrm{mm}$ & 0 \\
1\# AS-U to 7\# AS-U & symmetrical position \\
adjustment amount & AS-U control $+100 \%$ \\
\hline
\end{tabular}

The lateral thickness difference after rolling is shown in Figure 10. After the four groups of symmetrical position AS-U combinations control, there is local thickness depression near the $100 \mathrm{~mm}-300 \mathrm{~mm}$ area from the edge, which shows that the symmetrical position AS-U press adjustment cannot completely eliminate thickness depression in the high-order flatness defect area.
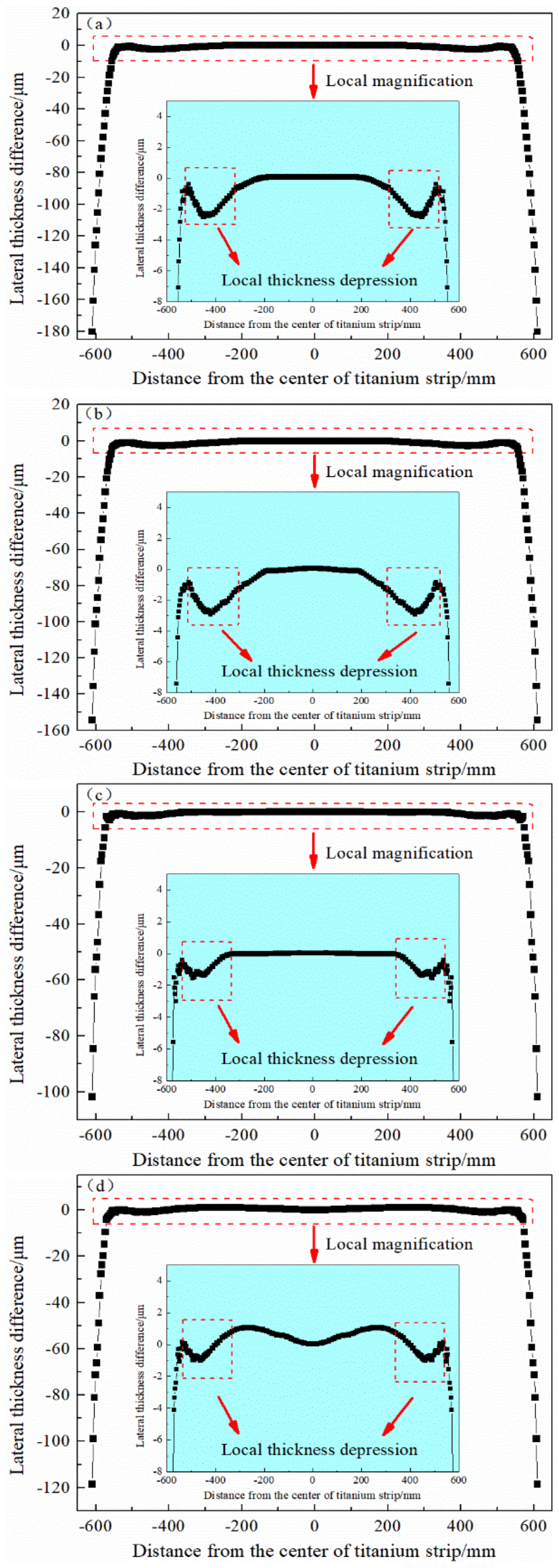
Figure. 10 Lateral thickness difference after rolling under AS-U press adjustment. (a) $1 \# \& 7 \#$ AS-U; (b) 2\#\&6\# AS-U; (c) 3\#\&5\# AS-U; (d) 4\# AS-U.

After the symmetrical position AS-U combination control, the value of depression $\gamma$ and the flatness value caused by the local depression $\lambda_{y}$ are in Table 9. After $1 \# \& 7 \#$ AS-U press adjustment, the value $\gamma$ and $\lambda_{\text {y }}$ increased by $17.6 \%$ and after $2 \# \& 6 \#$ AS-U press adjustment, the value $\gamma$ and $\lambda_{\gamma}$ increased by $29.4 \%$, 2\#\&6\# AS-U press adjustment will aggravate the high-order flatness defect more easily. After 3\#\&5\# AS-U and 4\# AS-U press adjustment, the value $\gamma$ and $\lambda_{y}$ decreased by $41.2 \%$.

In order to better grasp the symmetrical position AS-U press adjustment characteristics on high-order flatness defect area, it is also necessary to master the symmetrical position AS-U press adjustment characteristics on the full width of titanium strip. This study uses the lateral thickness difference between AS-U press adjustment and without the AS-U press adjustment as the variation of thickness profile, as shown in Figure 11. It can be founded that increasing 1\#\&7\# AS-U or 2\#\&6\# AS-U press adjustment will decrease significantly the thickness of the $0 \mathrm{~mm}-75 \mathrm{~mm}$ area from the edge and aggravate the thickness depression in the high-order flatness defect area. The thickness of 0 $\mathrm{mm}-75 \mathrm{~mm}$ away from the edge decreasing ability of 2\#\&6\# AS-U press adjustment is 2.6 times of that of 1\#\&7\# AS-U press adjustment. In addition, increasing 3\#\&5\# AS-U or 4\# AS-U press adjustment will increase significantly the thickness of the $0 \mathrm{~mm}-75 \mathrm{~mm}$ area from the edge and relieve the thickness depression in the highorder flatness defect area. The thickness of 0-75 mm away from the edge increasing ability of $3 \# \& 5 \#$ AS-U press adjustment is 1.6 times of that of $4 \#$ AS-U press adjustment. In the case of large tension in cold rolling, the increase of tensile stress at the edge is easy to cause strip breakage, 4\# AS-U press adjustment is more conducive to alleviate the high-order flatness defect.

Table 9. The $\gamma$ and the $\lambda_{y}$ after AS-U press adjustment.

\begin{tabular}{ccccc}
\hline Parameters & 1\#\&7\# & 2\#\&6\# & 3\#\&5\# & $4 \#$ \\
\hline$\gamma / \mu \mathrm{m}$ & 2.0 & 2.2 & 1.0 & 1.0 \\
$\lambda_{\gamma} / \mathrm{IU}$ & 165.4 & 182.0 & 82.6 & 82.6 \\
\hline
\end{tabular}
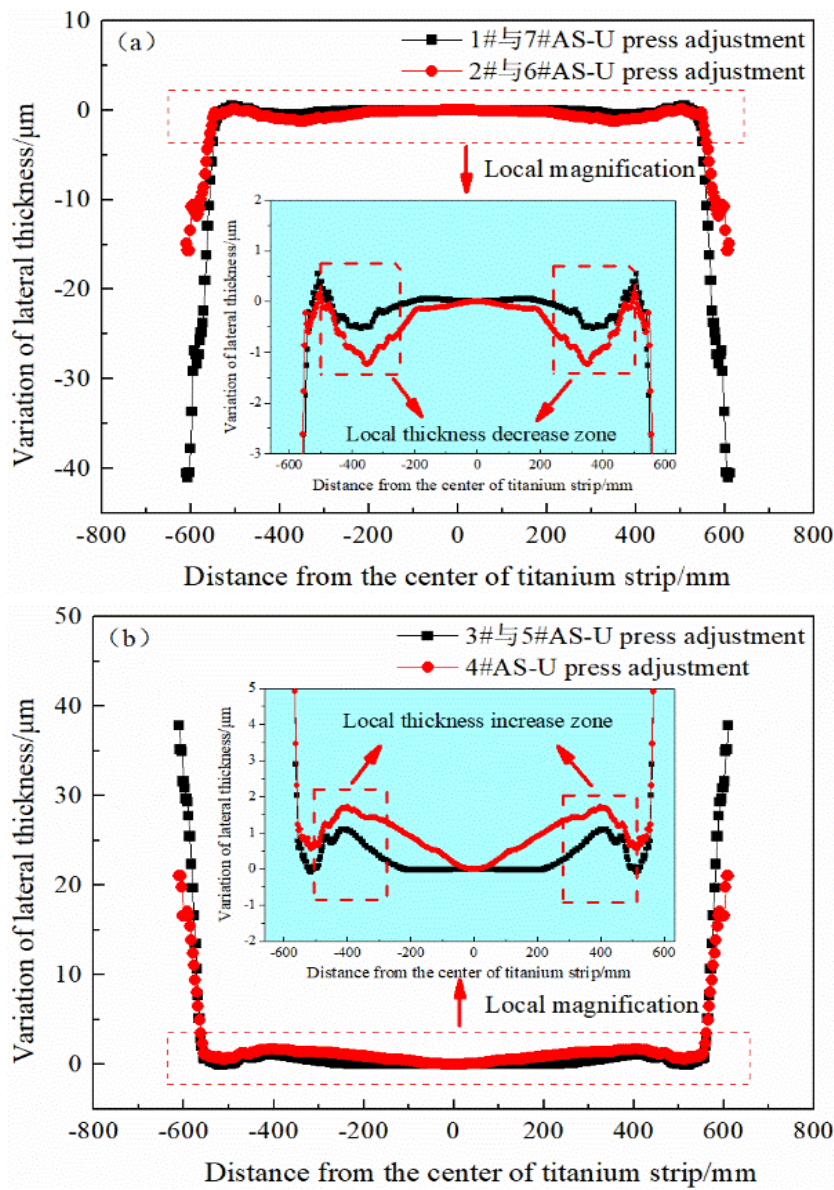

Figure. 11 Variation of section thickness under the press adjustment of symmetrical position AS-U. (a) $1 \# \& 7 \#$ AS-U or 2\#\&6\# AS-U; (b) 3\#\&5\# AS-U or $4 \#$ AS-U.

\subsection{Lateral thickness difference after rolling under roll shift adjustment}

The 1st intermediate taper roll can adjust the thickness of the edge area through online shifting. Therefore, the roll shifting adjustment is another important shape control method for a 20-high mill. This study analyzes the adjustment characteristics of the amount of roll shifting to the high-order flatness defect. The simulation conditions are as shown in Table 10.

Table 10. The simulation conditions under roll shift adjustment.

\begin{tabular}{cc}
\hline Parameters & Value \\
\hline Width strip/mm & 1250 \\
Entrance thickness/mm & 1.540 \\
Exit thickness/mm & 1.211 \\
Entrance tension/MPa & 70 \\
Exit tension/MPa & 130 \\
Amount of roll shift $/ \mathrm{mm}$ & $30 / 50 / 70$ \\
1\# AS-U to 7\# AS-U & $0 \%$ \\
\hline
\end{tabular}


adjustment amount

The lateral thickness difference after rolling is shown in Figure 12. After different amount of roll shift adjustment, there is local thickness depression near the $100 \mathrm{~mm}-300$ $\mathrm{mm}$ area from the edge, which shows that roll shift adjustment also cannot completely eliminate thickness depression in the high-order flatness defect area.
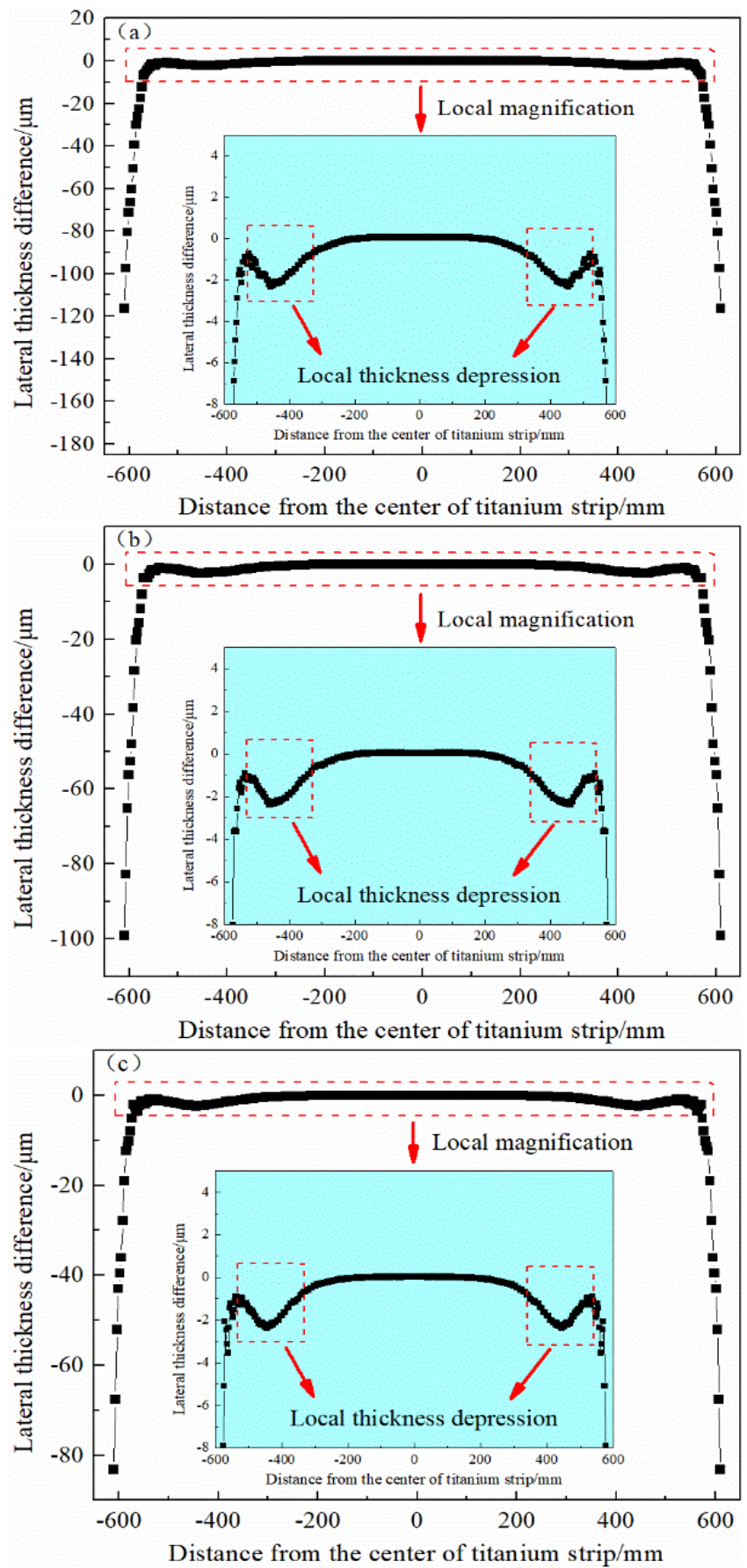

Figure. 12 Lateral thickness difference after rolling under roll shift adjustment. (a) roll shift $30 \mathrm{~mm}$; (b) roll shift $50 \mathrm{~mm}$; (c) roll shift $70 \mathrm{~mm}$.

After the roll shift adjustment, the value of depression $\gamma$ and the flatness value caused by the local depression $\lambda_{y}$ are in Table 11. It can be found that there is no obvious change in the value of depression under different amount of roll shifting and the roll shift adjustment cannot effectively alleviate high-order flatness defect.

Table 11. The $\gamma$ and the $\lambda_{y}$ after roll shift adjustment.

\begin{tabular}{cccc}
\multirow{2}{*}{ Parameters } & \multicolumn{3}{c}{ Amount of roll shift $/ \mathrm{mm}$} \\
\cline { 2 - 4 } & 30 & 50 & 70 \\
$\gamma / \mu \mathrm{m}$ & 1.7 & 1.8 & 1.7 \\
$\lambda_{\gamma} / \mathrm{IU}$ & 140.6 & 148.9 & 140.6
\end{tabular}

In order to compare the thickness change across the strip width after roll shift adjustment, this study uses the lateral thickness difference between the roll shift adjustment and without the roll shift adjustment as the variation of thickness profile, as shown in Figure 13. It can be founded that increasing amount of roll shift will increase significantly the thickness of the $0 \mathrm{~mm}-75 \mathrm{~mm}$ area from the edge. After the roll shift adjustment of $30 \mathrm{~mm}, 50 \mathrm{~mm}$ and $70 \mathrm{~mm}$, the thickness of $0 \mathrm{~mm}-75 \mathrm{~mm}$ away from the edge will be increased $22.3 \%, 38.9 \%, 53.1 \%$ respectively. But there is no obvious change in the thickness of the other area.

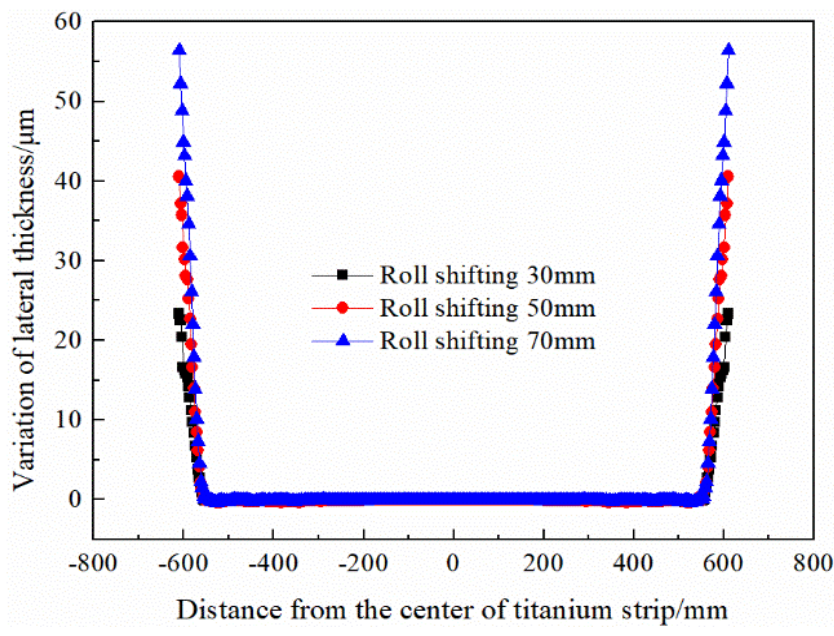

Figure. 13. Variation of section thickness under roll shift adjustment.

\subsection{Industry test of 4\# AS-U press adjustment characteristics}

This study uses the 20-high mill FEM model to analysis the adjustment characteristics of the high-order flatness defect by two adjustment methods, and finds that the $3 \# \& 5 \#$ AS-U or $4 \#$ AS-U press adjustment can alleviate the local thickness depression in the high-order flatness defect area and thus reduce the compressive stress in the 
high-order flatness defect area. In addition, when increasing 4\# AS-U press adjustment, the thickness of the range of $0 \mathrm{~mm}-75 \mathrm{~mm}$ from the edge will increase and the thickness of the range of $200 \mathrm{~mm}$ away from the center point will decrease. In other words, after $4 \mathrm{v} \#$ control, the change value of the shape stress in the $0 \mathrm{~mm}-75 \mathrm{~mm}$ area develops in the direction of tensile stress, and the shape stress in the area $200 \mathrm{~mm}$ away from the center point develops in the direction of compressive stress. The industry test parameters are shown in table 12.

Table 12. Industry test condition of 4 \# AS-U adjustment.

\begin{tabular}{cc}
\hline Parameters & Value \\
\hline Width strip/mm & 1250 \\
Entrance thickness/mm & 2.850 \\
Exit thickness/mm & 2.323 \\
Entrance tension/MPa & 70 \\
Exit tension/MPa & 120 \\
Amount of roll shift/mm & 46.8 \\
AS-U adjustment amount & $16 \% 、-18 \% 、 19 \%$ 、- \\
in other positions & $16 \% 、-1 \% 、 22 \%$ \\
4\# AS-U adjustment & $-3 \%$ \\
amount before test & \\
4\# AS-U adjustment & \\
amount after test & $+11 \%$ \\
\hline
\end{tabular}

The stress distribution on the shape meter before and after the industry test is shown in Figure 14. It can be found that the stress distribution has changed after increasing $4 \#$ AS-U press adjustment. The variation of stress distribution after 4\# AS-U press adjustment is shown in Figure 15. The thickness decreases in the range of $200 \mathrm{~mm}$ from the center point, leading the stress distribution in this area to develop in the direction of compressive stress, simultaneously, the thickness of the high-order flatness defect area and the range of $0 \mathrm{~mm}-75 \mathrm{~mm}$ from the edge increases, leading the stress distribution in this area to develop in the direction of tensile stress. It shows that $4 \#$ AS-U press adjustment can alleviate high-order flatness defect to a certain extent. In addition, it also shows that the FEM model result can be highly consistent with the industry test result.
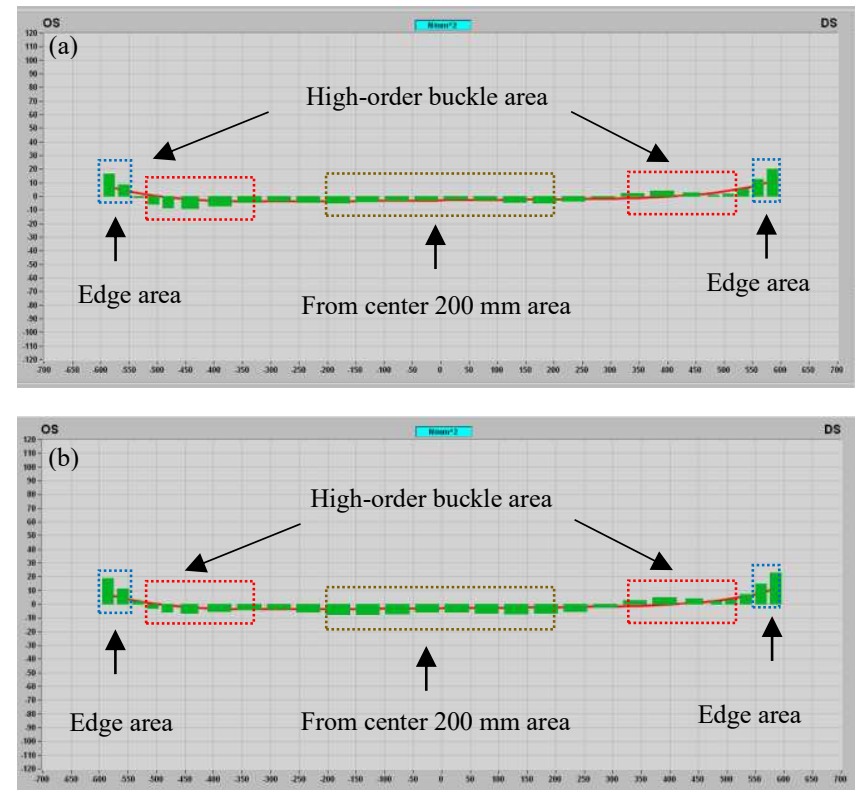

Figure. 14 4\# AS-U adjustment test. (a) before industry test; (b) after industry test.

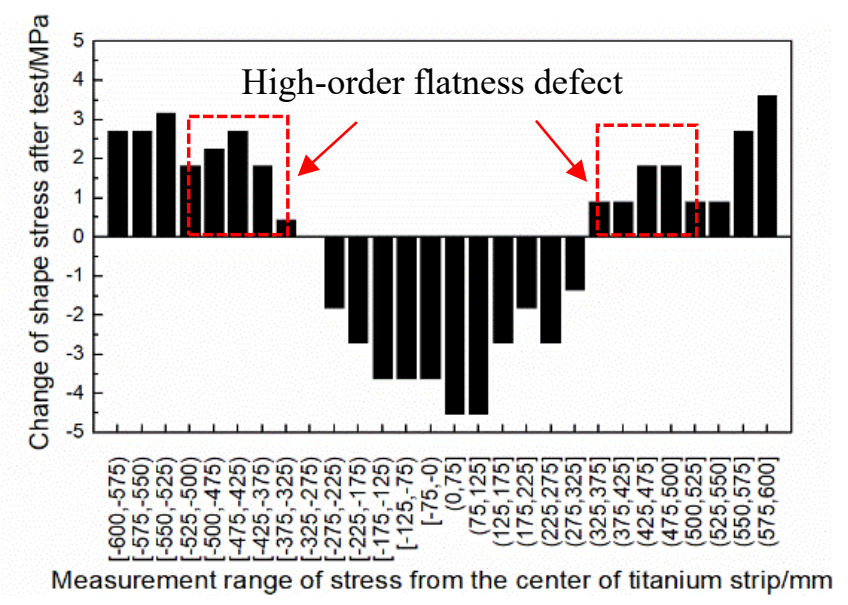

Figure. 15 The variation of stress distribution after industry test.

\section{Conclusions}

(1) Based on ABAQUS finite element software, an implicit integration calculation model of rolls-strip for 20high mill is established, which considers the anisotropic mechanical characteristics of commercially pure titanium and can realize dynamic rolling process. By comparing with the industry data, the thickness deviation of the section was $0.65 \%$ and the rolling force deviation was $8.18 \%$.

(2) The FEM model is used to calculate the adjustment characteristics of high-order flatness defect on two shape adjustment methods of 20-high mill, and it is found that the adjustment of roll shift cannot effectively control the highorder flatness defect, that $1 \# \& 7 \#$ AS-U or $2 \# \& 6 \#$ AS-U 
press adjustment will increase the degree of high-order flatness defect, and that $3 \# \& 5 \#$ AS-U or $4 \#$ AS-U can alleviate the degree of high-order flatness defect to a certain extent.

(3) According to the industry test, the compressive stress in the high-order flatness defect decreased after increasing the 4\# AS-U press adjustment amount. The industry test result is consistent with the FEM model result, further verifying the high reliability of the 20-high mill model in this study.

\section{Availability of data and materials}

Not applicable

\section{Acknowledgements}

The authors would like to express sincere gratitude to the National Engineering Research Center for Advanced Rolling Technology for providing the simulation equipment and the Hunan Xiangtou Goldsky Titanium Metal Co. , Ltd for providing the test opportunity.

\section{Authors' contributions}

GZ is responsible for writing the entire paper and conducting the simulation model. HL provided advice on the abstract. AH supervised the entire paper. CL and SC checked the validation results. WS provided advice on the conclusion. $\mathrm{ZL}$ and $\mathrm{CH}$ guided the industrial test. All authors read and approved the final manuscript.

\section{Funding}

The authors gratefully acknowledge the support from Guangxi Special Funding Programme for InnovationDriven Development (GKAA17202008) and the National Natural Science Foundation of China (No. 51674028, No. 52004029).

\section{Competing interests}

The authors declare that they have no competing interests.

\section{References}

[1] Liu, D.K., Huang, G.S., Gong, G.L., Wang, G.G., Pan, F.S. (2017). Influence of different rolling routes on mechanical anisotropy and formability of commercially pure titanium sheet. Transactions of Nonferrous Metals Society of China, 27(6), 13061312.

[2] LIU, N., Wang, Y., He, W.J., Li, J., Chapuis, A., Luan, B.F., LIU, Q. (2018). Microstructure and textural evolution during cold rolling and annealing of commercially pure titanium sheet. Transactions of
Nonferrous Metals Society of China, 28(6), 1123 1131.

[3] Roth, A., Lebyodkin, M.A., Lebedkina, T.A., Lecomte, J.S., Richeton, T., Amouzou, K.E.K. (2014). Mechanisms of anisotropy of mechanical properties of $\alpha$-titanium in tension conditions. Materials Science \& Engineering A, 596, 236-243.

[4] Zhu, X., Feng, G.H., Zhang, H.L. (2014). Defect analysis on ridge buckles of cold-rolled Pure titanium strip. Hot Working Technology, 43(19), 227-230.

[5] Shi, Y.M., Li, Z.M., Cao, Z.Y., Zhang, L.Y., Zhang, Q.D. (2017). Simulation on transverse stress distribution of titanium strips in steel strip continuous annealing furace. China Metallurgy, 27(9), 25-30.

[6] Wang, C.T., Fang, K.L. (2004). Shape control of sendzimir mill. Journal of Wuhan University of Science and Technology, 22(1), 8-11.

[7] Yu, H.L., Liu, X.H., Lee, G.T. (2007). Contact element method with two relative coordinates and its application to prediction of strip profile of a sendzimir mill. ISIJ International, 47(7), 996-1005.

[8] Zhang, Q.D., Dai, C., Wen, J., Zhang, X.F., Qin, J. (2013). Simulation and analysis on shape control behavior of 20-h sendzimir mill, Steel Rolling, 30(3), 1-6.

[9] Yuan, Z.W., Xiao, H. (2015). Plate shape control theory and experiment for 20-high Mill. Journal of Iron and Steel Research International, 22(11), 9961001.

[10] Zhang, L.J., Zhang, Q.D., Yu, M. (2018). Analysis on shape control behavior of $20 \mathrm{~h}$ sendzimir mill by finite element method. Metallurgical Equipment, 000(1), 40-43

[11] Li, H.B., Zhen, Z.W., Dong, D.W., Han, G.M., Zhang, J., Hai, H.C., You, X.C. (2018). Edge-drop control behavior for silicon strip cold rolling with a sendzimir mill. Metals - Open Access Metallurgy Journal, 8(10), 783.

[12] Hara, K., Yamada, T., Takagi, K. (1991). Shape controllability for quarter buckles of strip in 20-high sendzimir mills. ISIJ International, 31(6), 607-613.

[13] Kim, J.T., Yi, J.J., Han, S.Y. (1996). Shape control of alloy steel rolled by sendzimir mill. Journal of Mechanical Science and Technology, 10(3), 277-285. 
[14] Aizawa, A., Kubo, T., Hara, K., Uchihata, O. (2006). Simulation model for predicting strip shape in consideration of characteristics of flexible backup rolls: development of controlling technology for strip shape in 20-high sendzimir mill. Journal of the Japan Society for Technology of Plasticity, 47(548), 845849.

[15] Lin, Z., Liu, M. (2009). Y elastic constants of polycrystalline materials with hexagonal system structure. Acta Physica Sinica, 58 (12), 8511-8521.

[16] Wang, C.Q., Shang, S.L., Wang, N. (2000). Calculation of elastic properties of metals and alloys with a hexagonal structure, The Chinese Journal of Nonferrous Metals, 10(2), 221-224.

[17] Toussaint, F., Tabourot, L., Ducher, F. (2008). Experimental and numerical analysis of the forming process of a CP titanium scoliotic instrumentation. Journal of Materials Processing Tech, 197(1-3), 1016.
[18] Xue, X.Y., Chen, J.Y., Li, H.W., Gao, E.Z., Li, J.S., Zhou, L. (2010). Anisotropic effects on cold deep drawing of TA1 spherial part, Journal of Plasticity Engineering, 17(2),11-16.

[19] Wang, L.F., Zhang, H., Huang, G.S., Cao, M., Cao, X.Q. Mostaed, E., Vedani, M. (2016). Formability and anisotropy of the mechanical properties in commercially pure titanium after various routes normal and different speed rolling. Journal of Materials Research, 31(21), 3372-3380.

[20] Pan, C.J. (2003). Production of 20 high rolling mill and high precision cold rolled steel strip. Beijing: Metallurgical Industry Press.

[21] Zhao, J.W., Wang, X.C., Yang, Q., Wang, Q.N., Wang, Y.Y., Li, W.P. (2020). Mechanism of lateral metal flow on residual stress distribution during hot strip rolling. Journal of Materials Processing Technology. doi.org:10.1016/j.jmatprotec.2020.116838. 
Figures

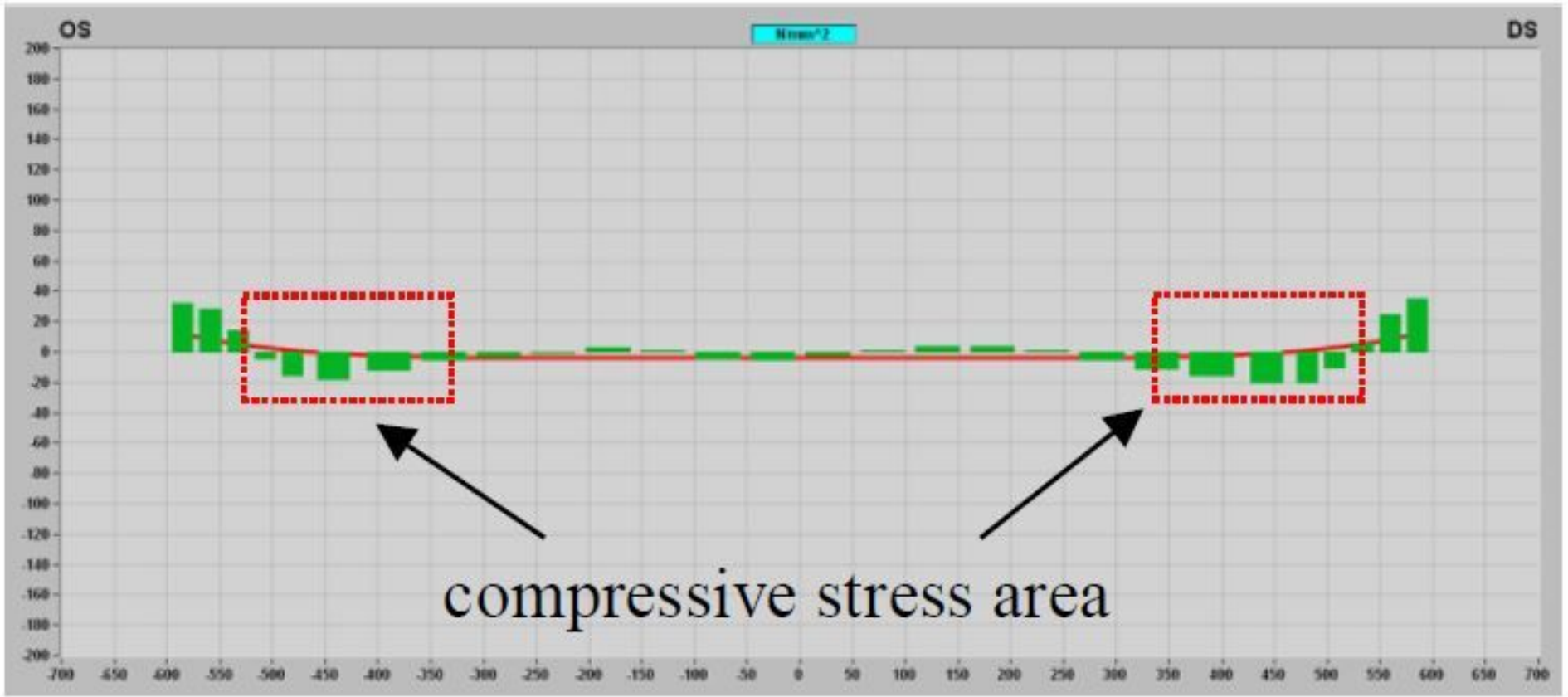

Figure 1

Stress distribution on the shape meter. 

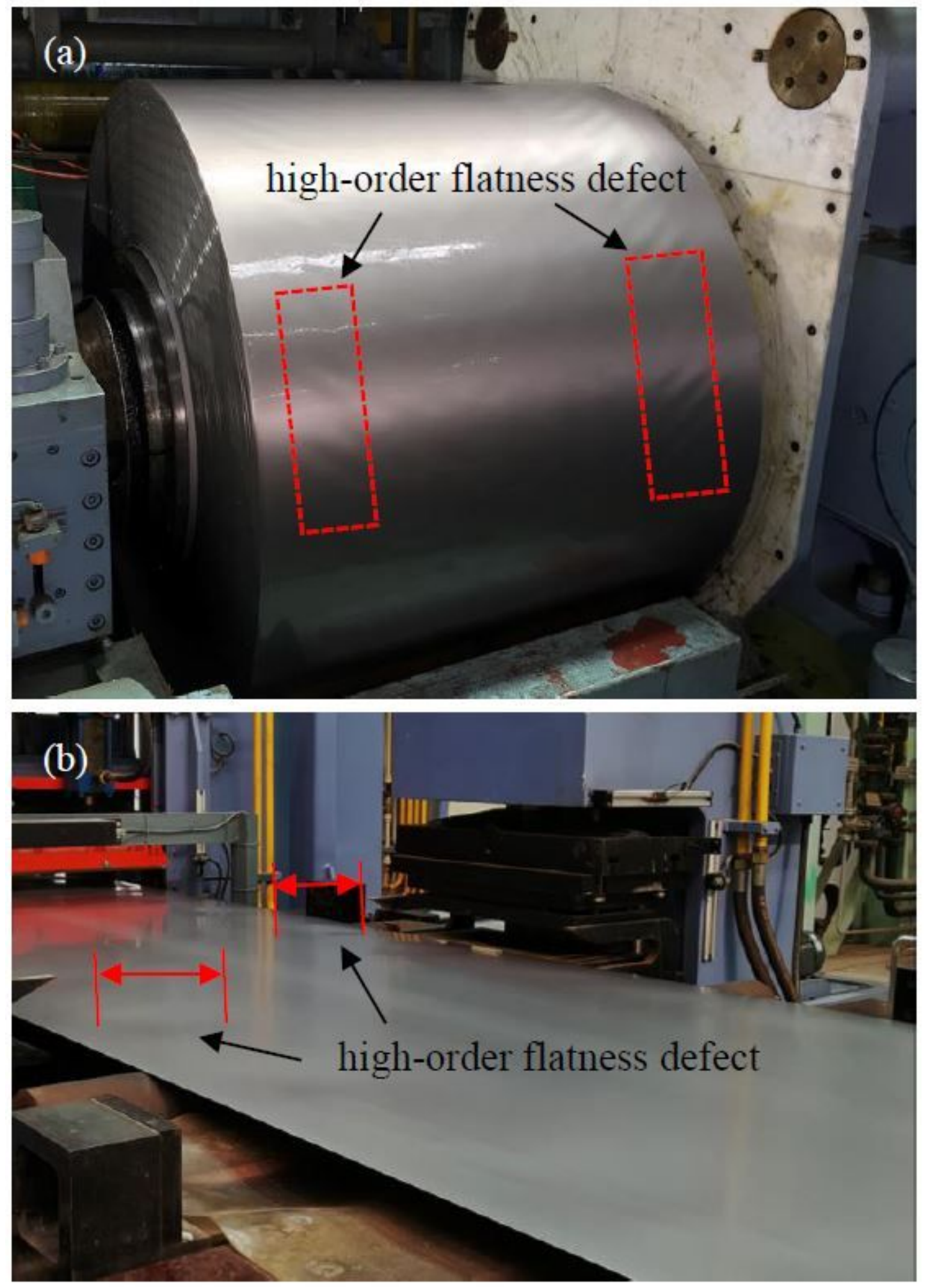

Figure 2

High-order flatness defect. (a) during coiling; (b) during straightening. 

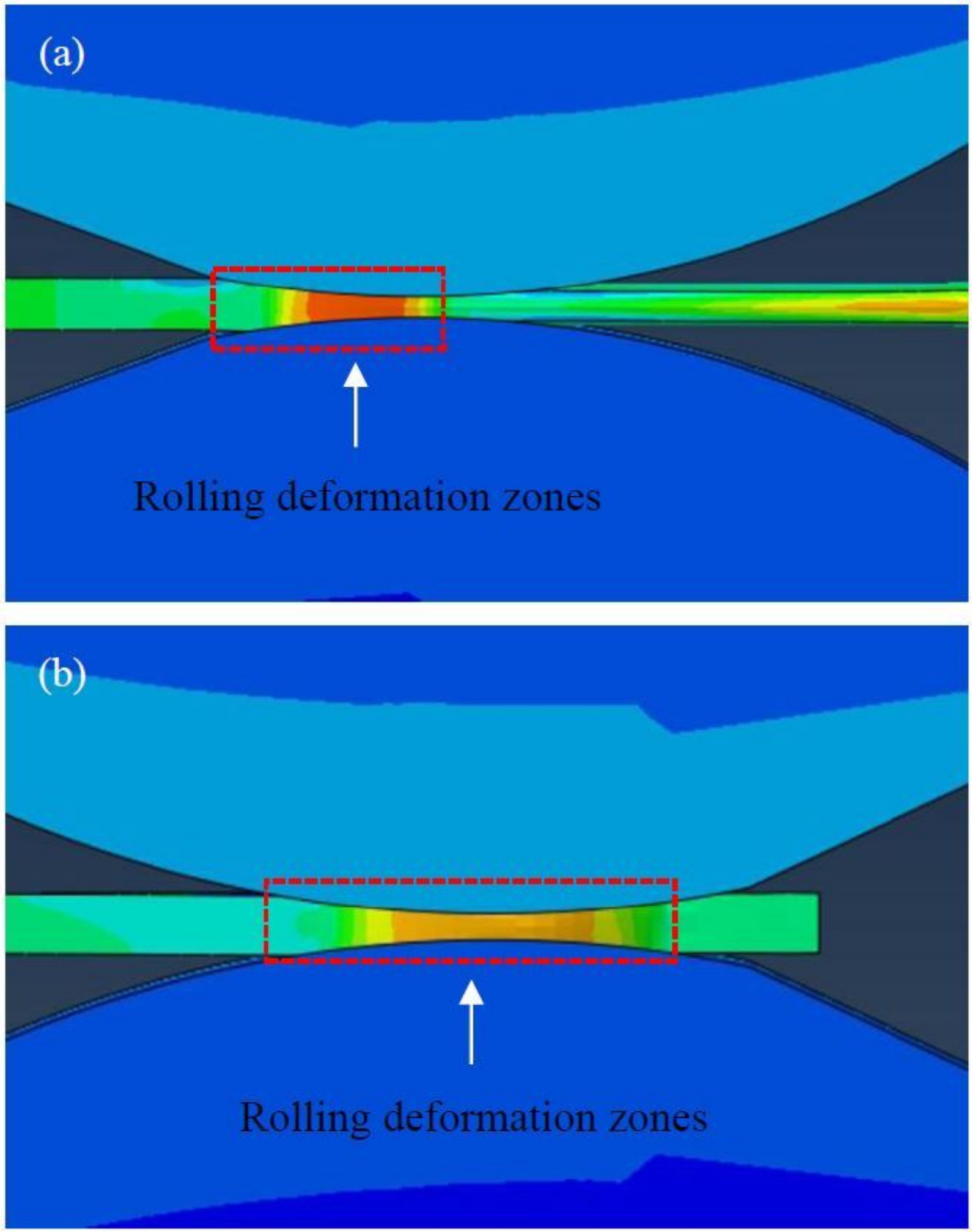

Figure 3

Rolling deformation area. (a) under rotation model; (b) under static model. 


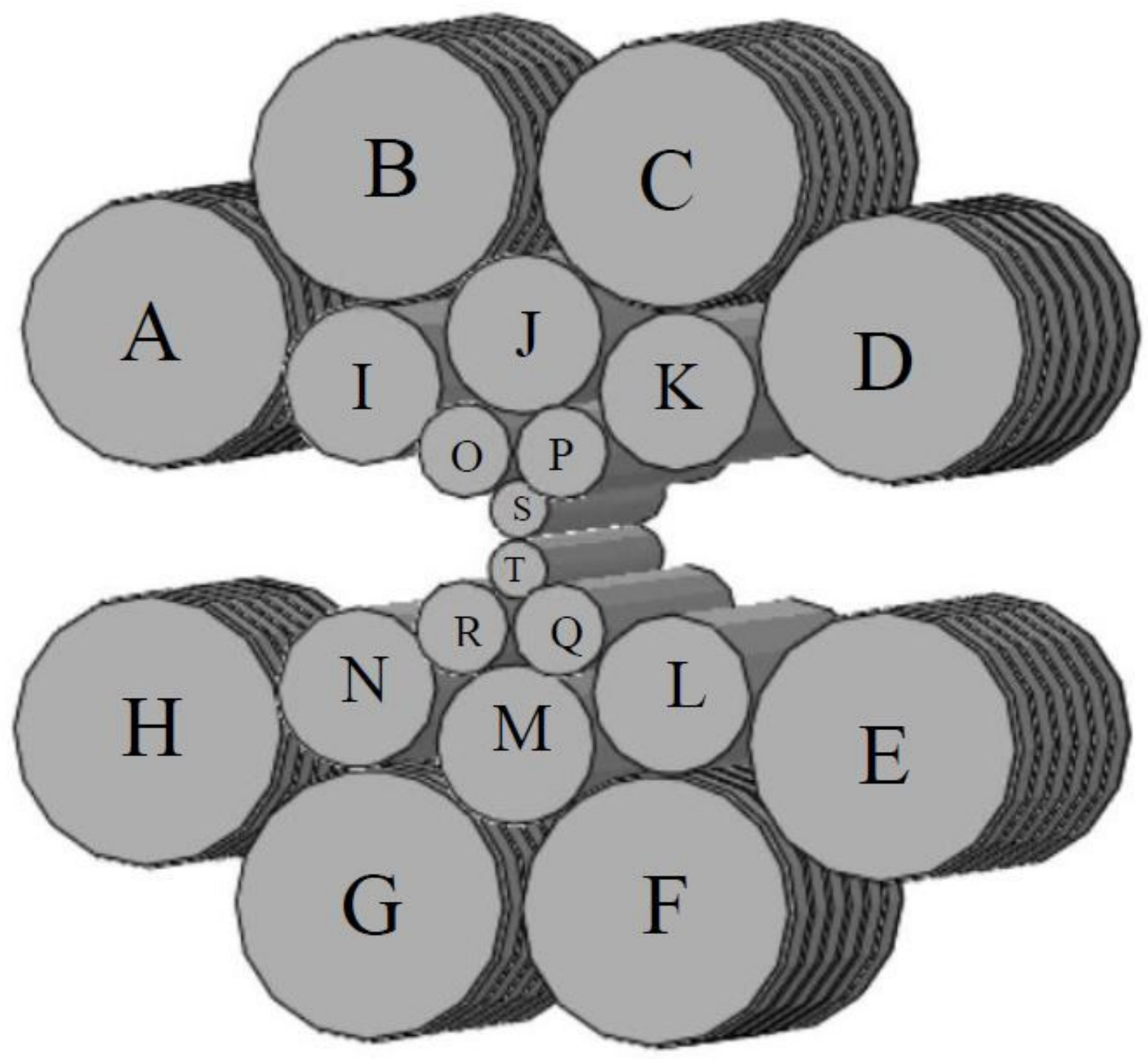

Figure 4

Roll system structure of 20-high mill. 


\section{Operation side}

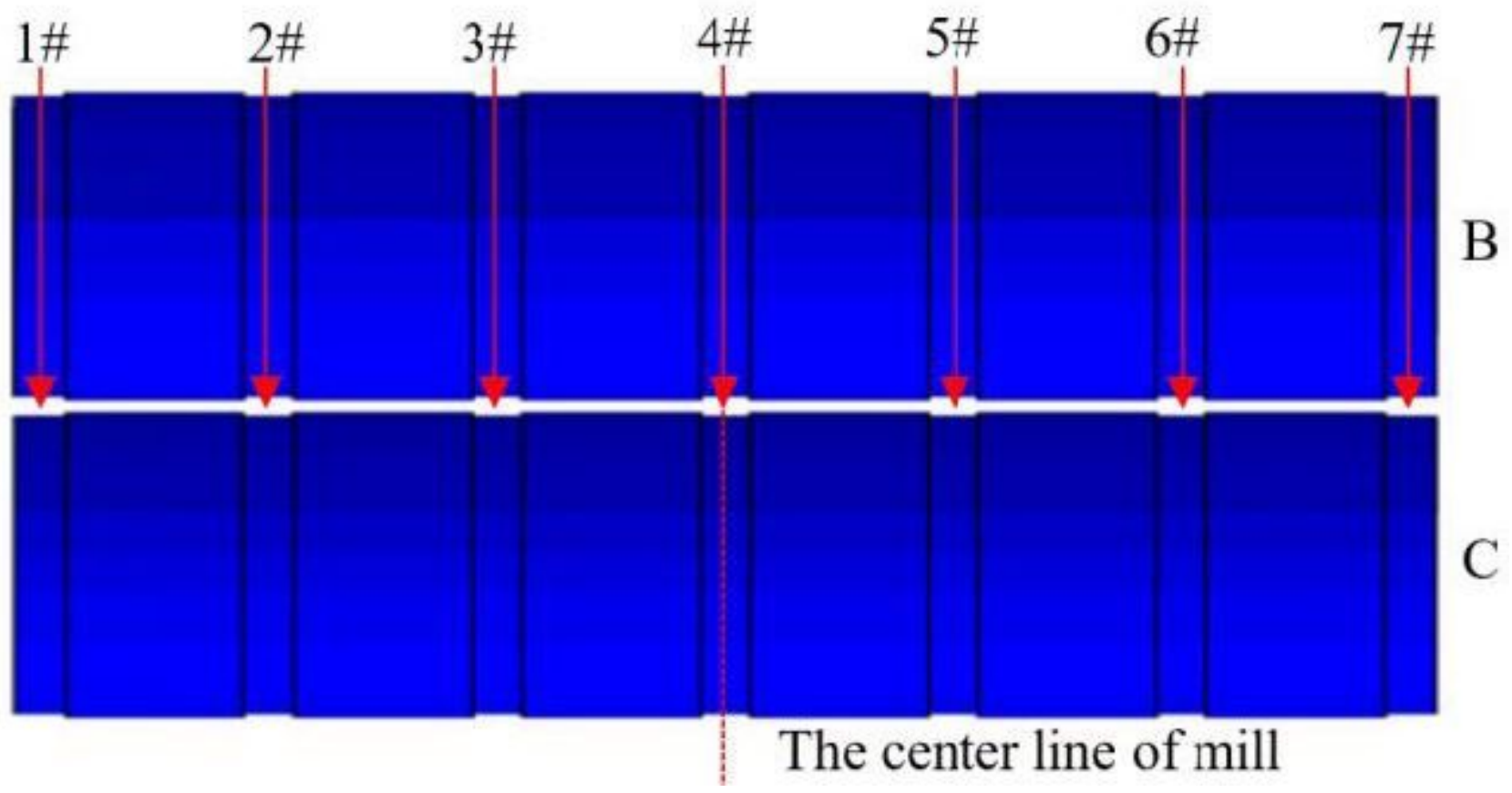

Figure 5

The AS-U adjustment means of 20-high mill.

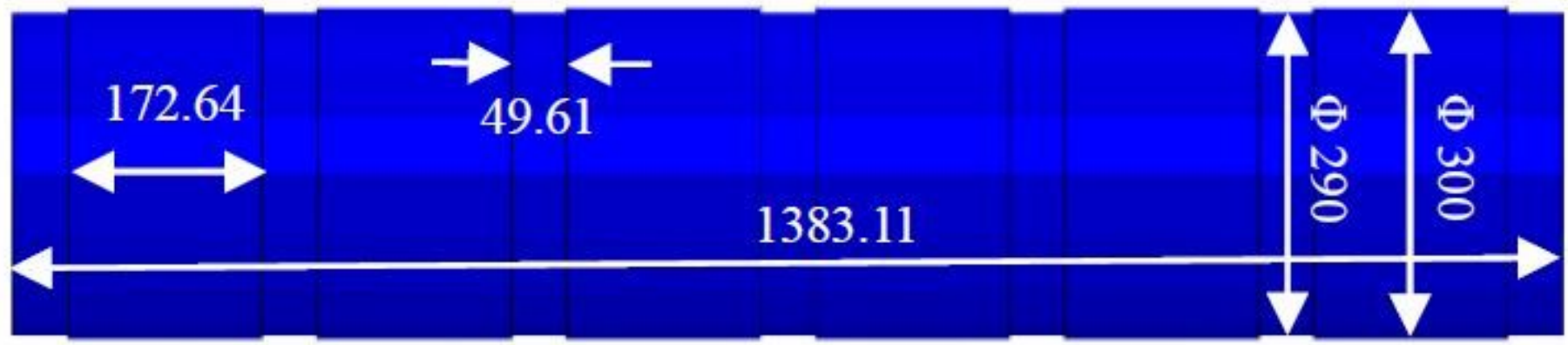

Figure 6

The simplified structure of backup roll. 


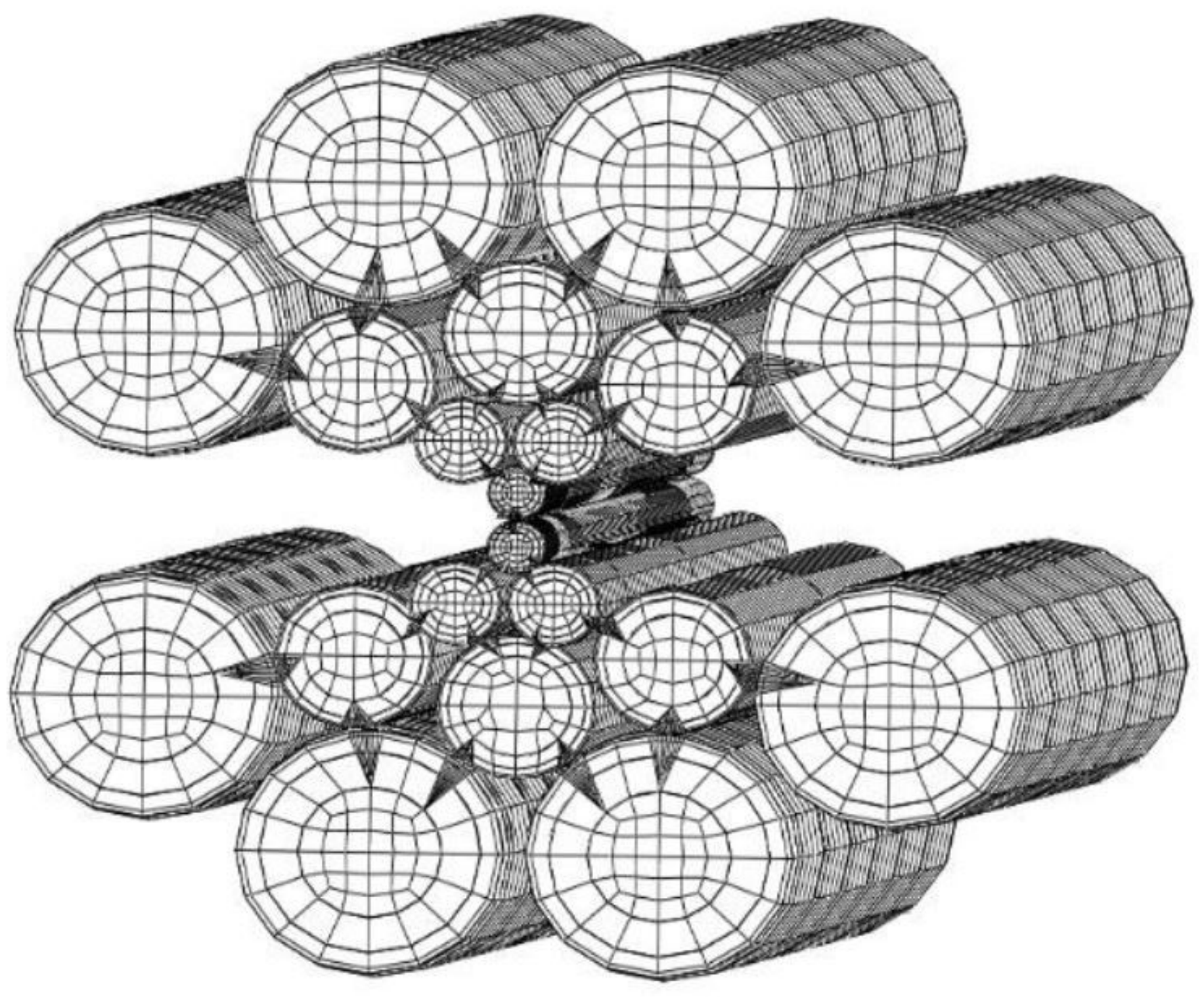

Figure 7

The FEM model of 20-high mill with the mesh refinement near the contact area. 


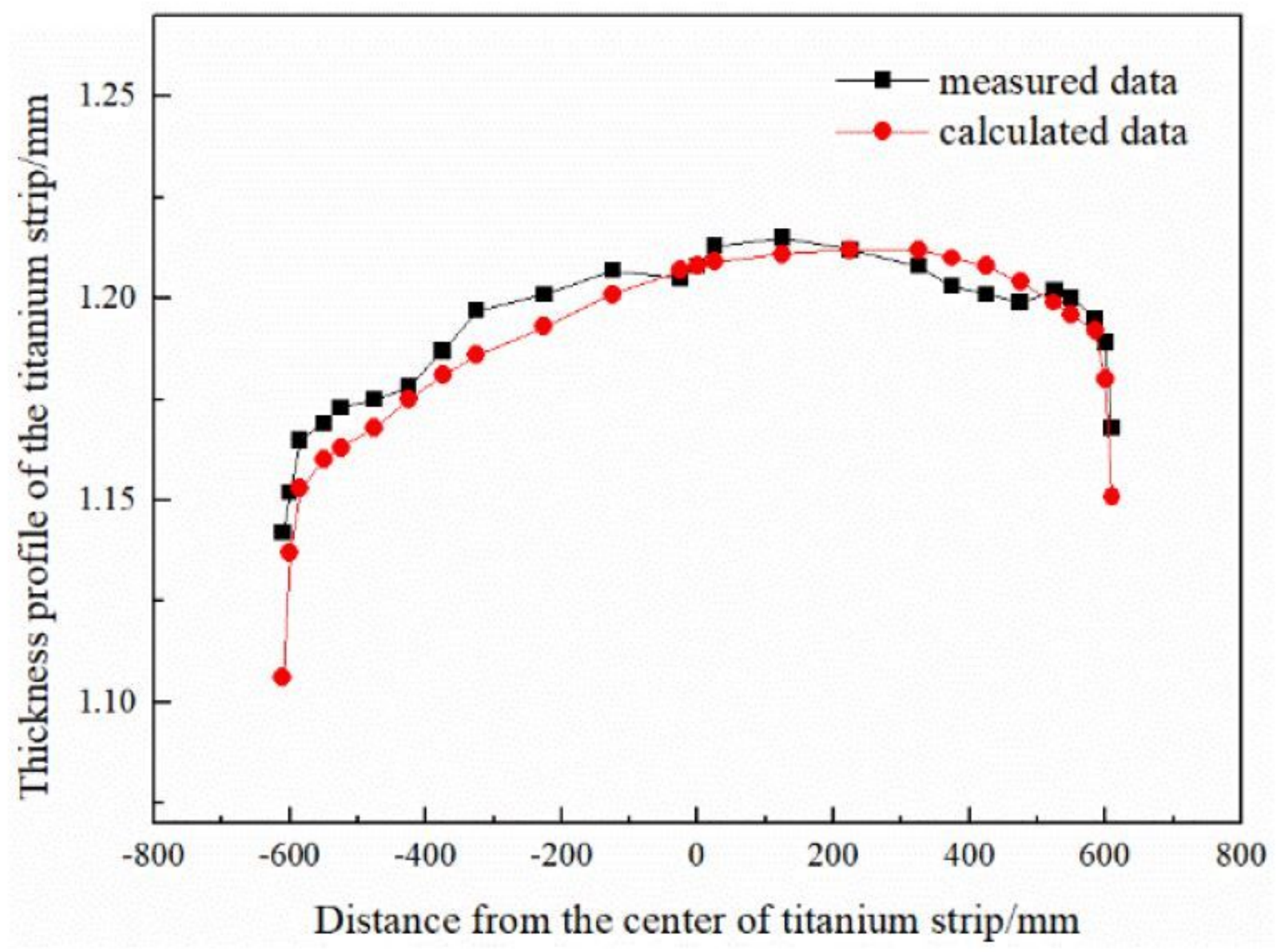

Figure 8

The comparison of measured and simulated thickness profiles. 


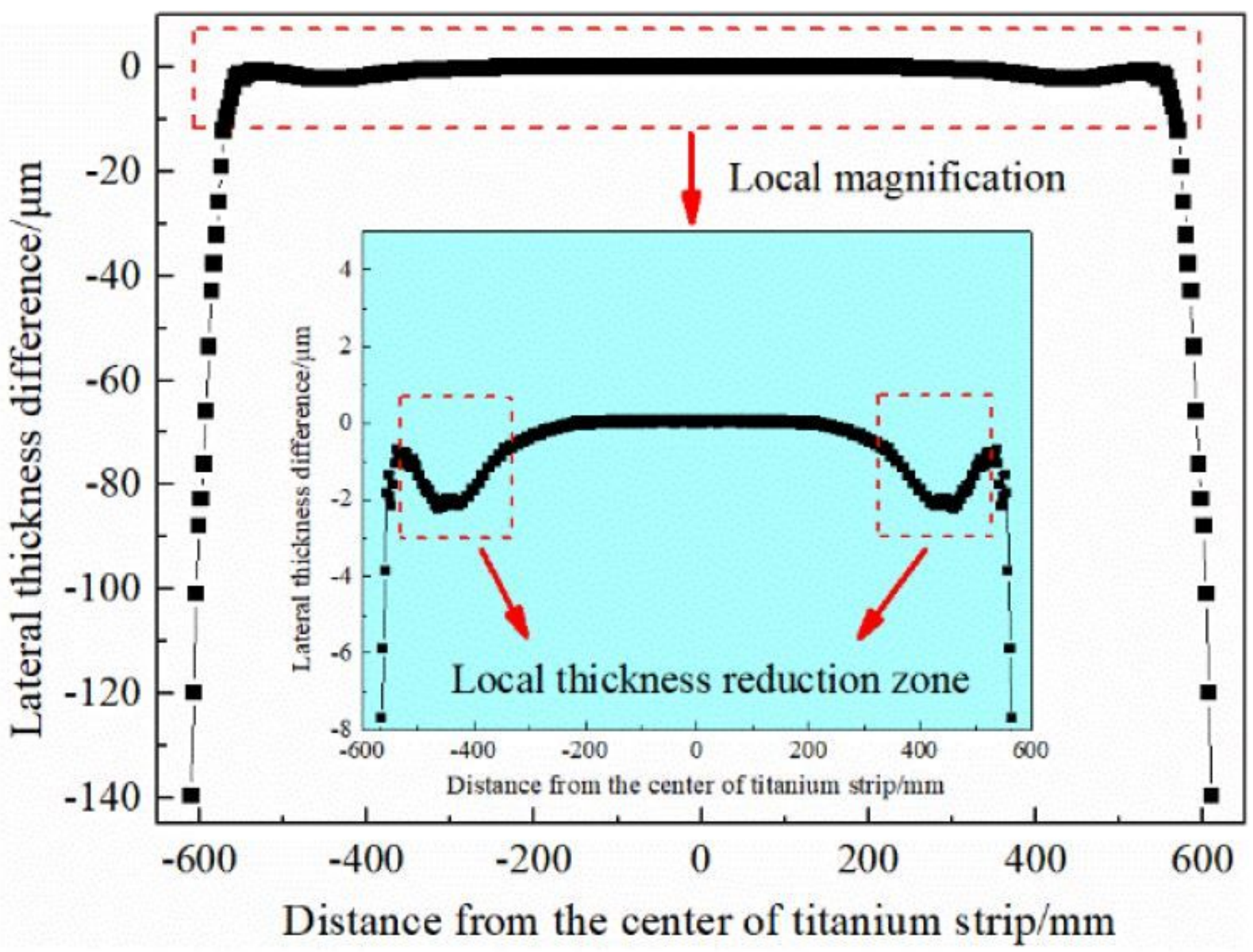

Figure 9

Lateral thickness difference after rolling without shape adjustment. 

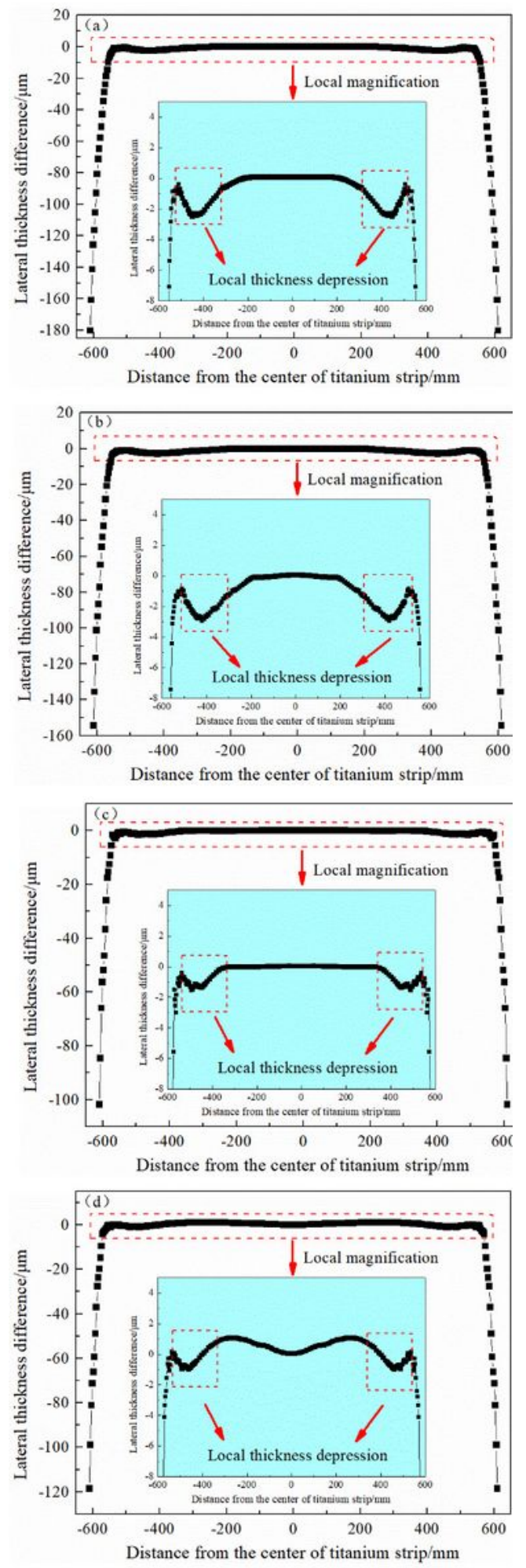

\section{Figure 10}

Lateral thickness difference after rolling under AS-U press adjustment. (a) 1\#\&7\# AS-U; (b) 2\#\&6\# AS-U; (c) 3\#\&5\# AS-U; (d) 4\# AS-U. 

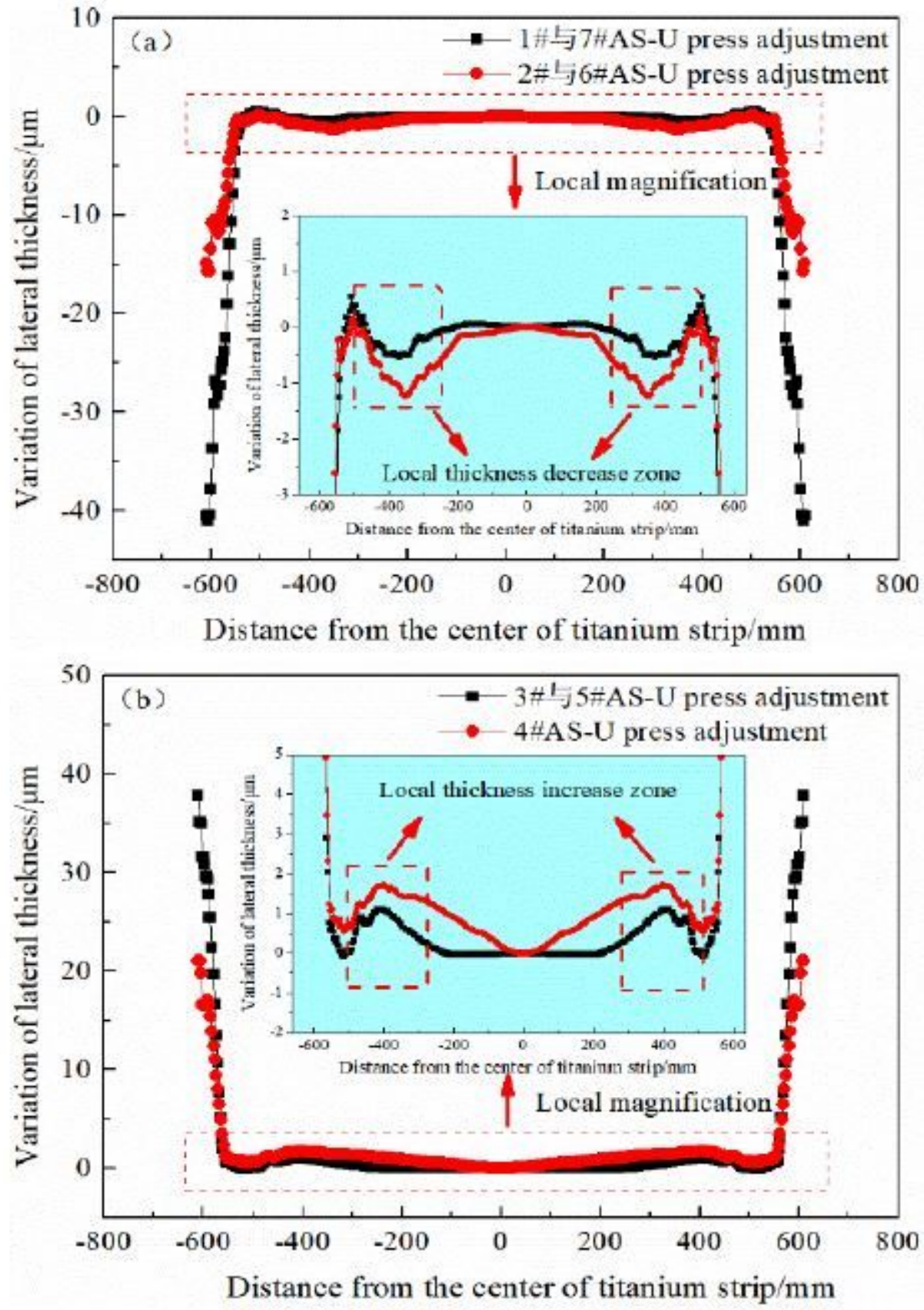

\section{Figure 11}

Variation of section thickness under the press adjustment of symmetrical position AS-U. (a) 1\#\&7\# AS-U or 2\#\&6\# AS-U; (b) 3\#\&5\# AS-U or 4\# AS-U. 

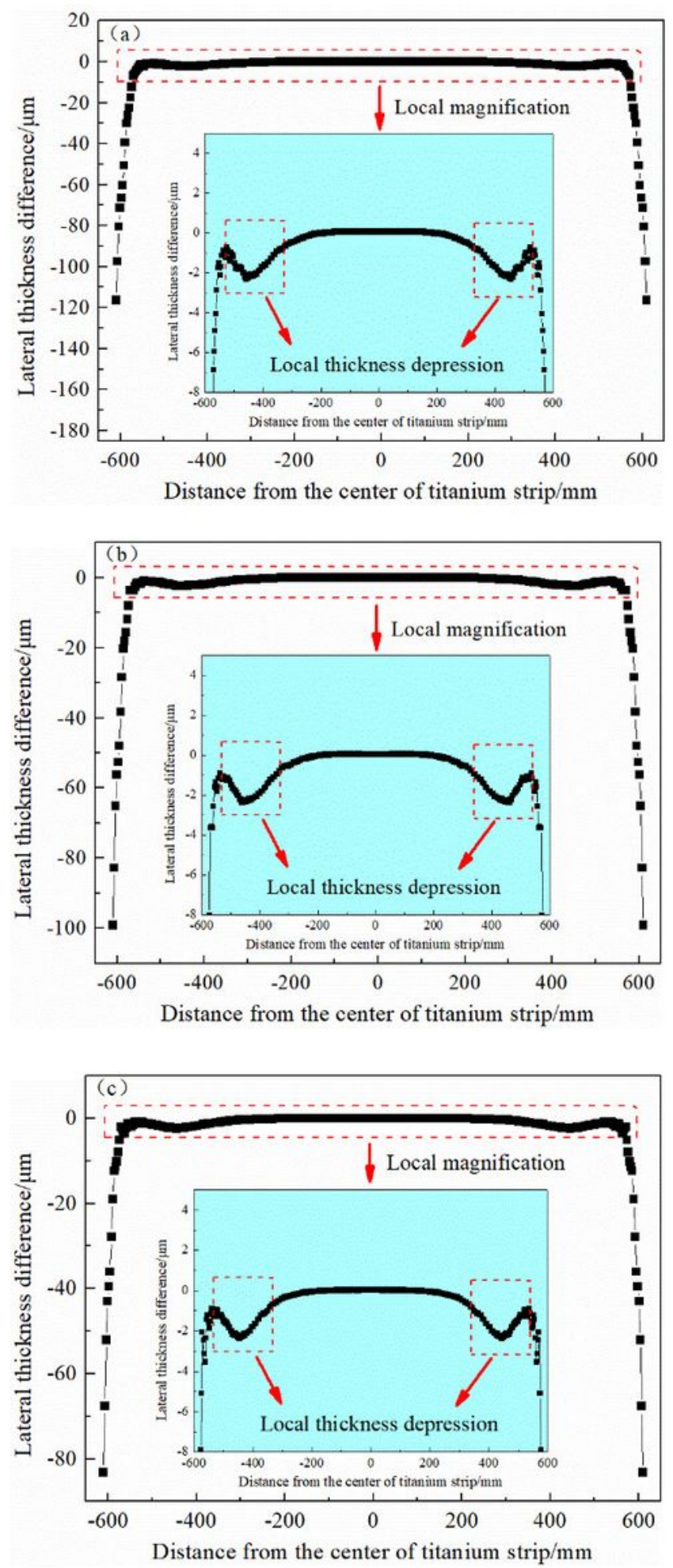

\section{Figure 12}

Lateral thickness difference after rolling under roll shift adjustment. (a) roll shift $30 \mathrm{~mm}$; (b) roll shift 50 $\mathrm{mm}$; (c) roll shift $70 \mathrm{~mm}$. 


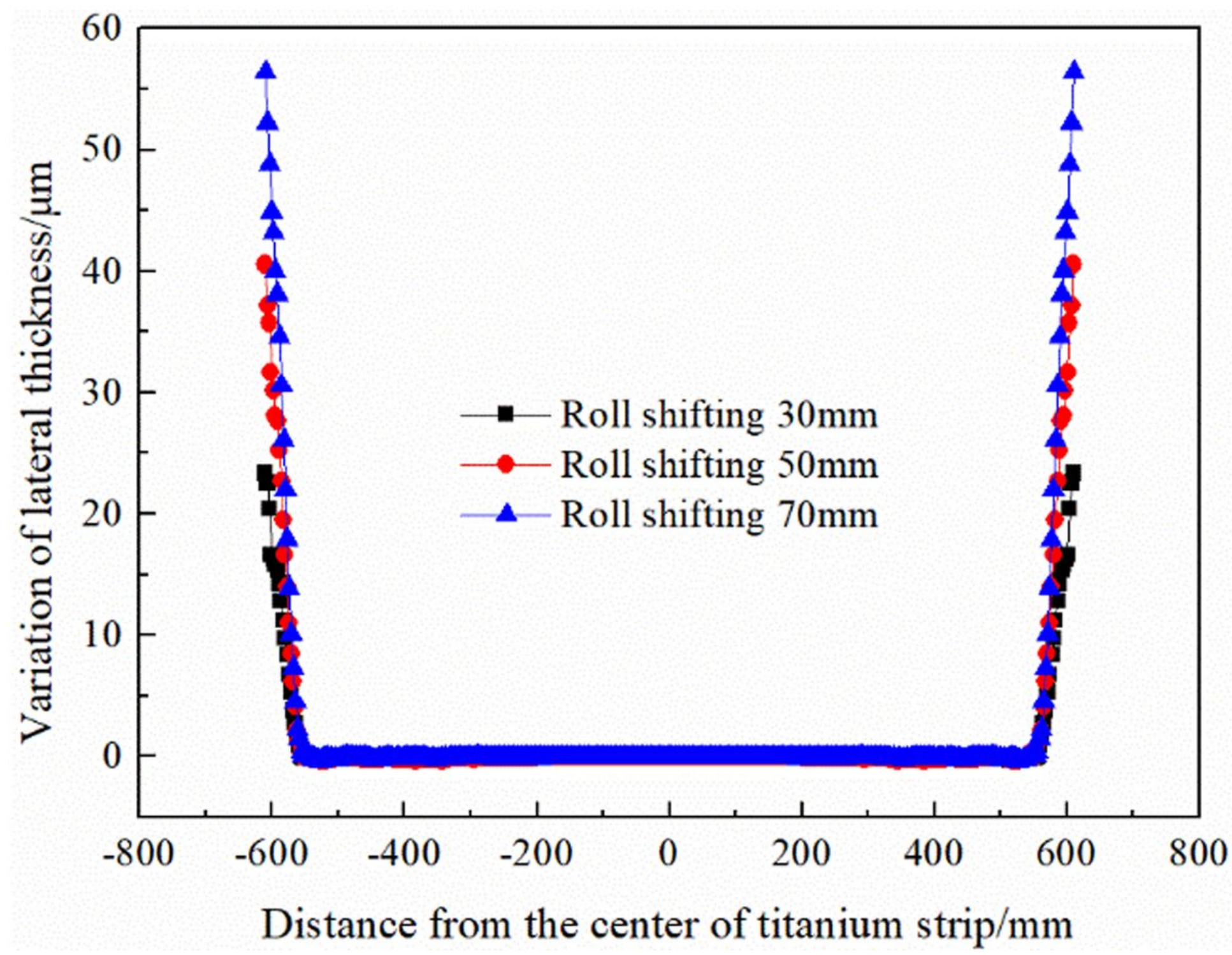

Figure 13

Variation of section thickness under roll shift adjustment. 

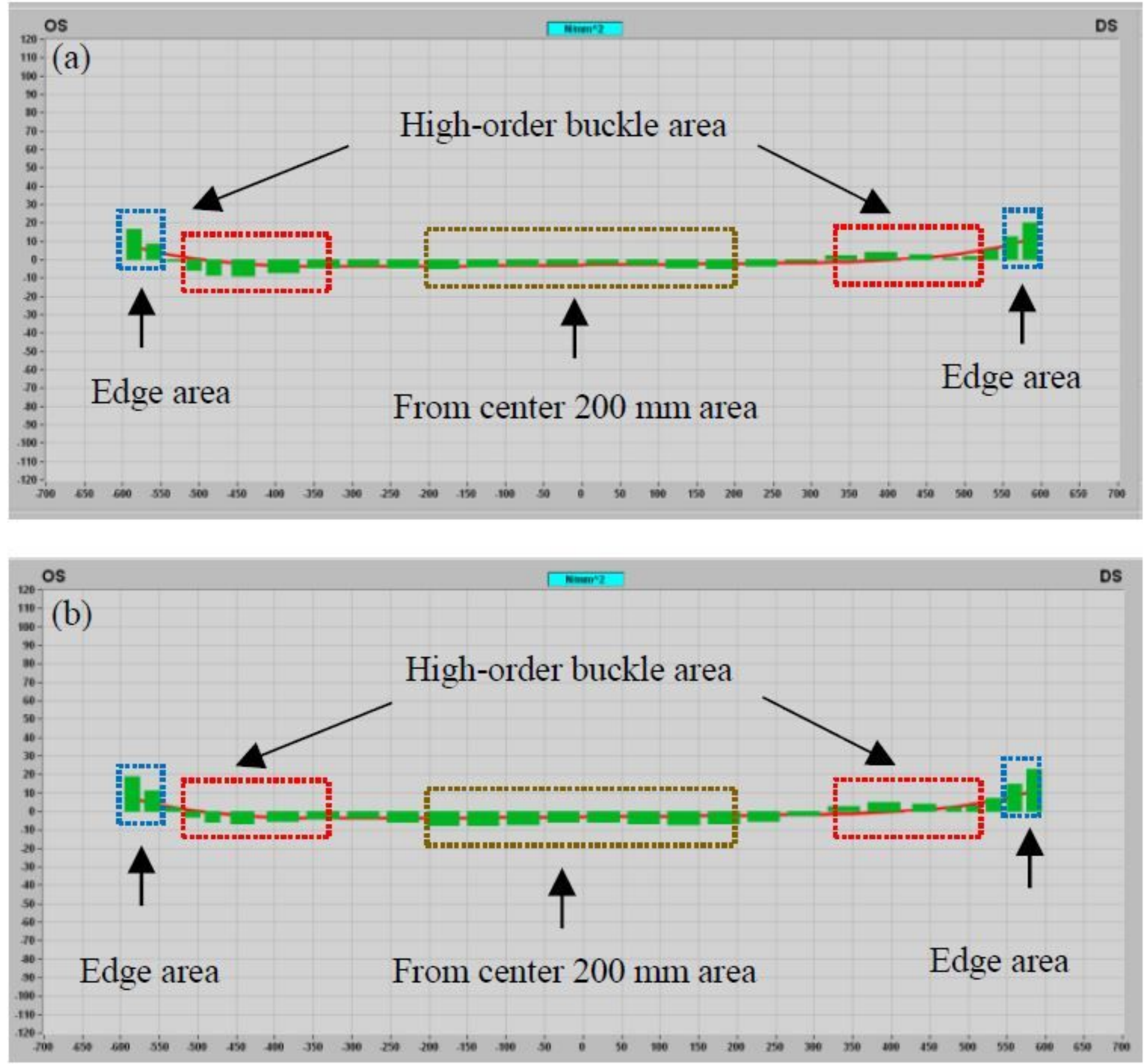

Figure 14

4\# AS-U adjustment test. (a) before industry test; (b) after industry test. 


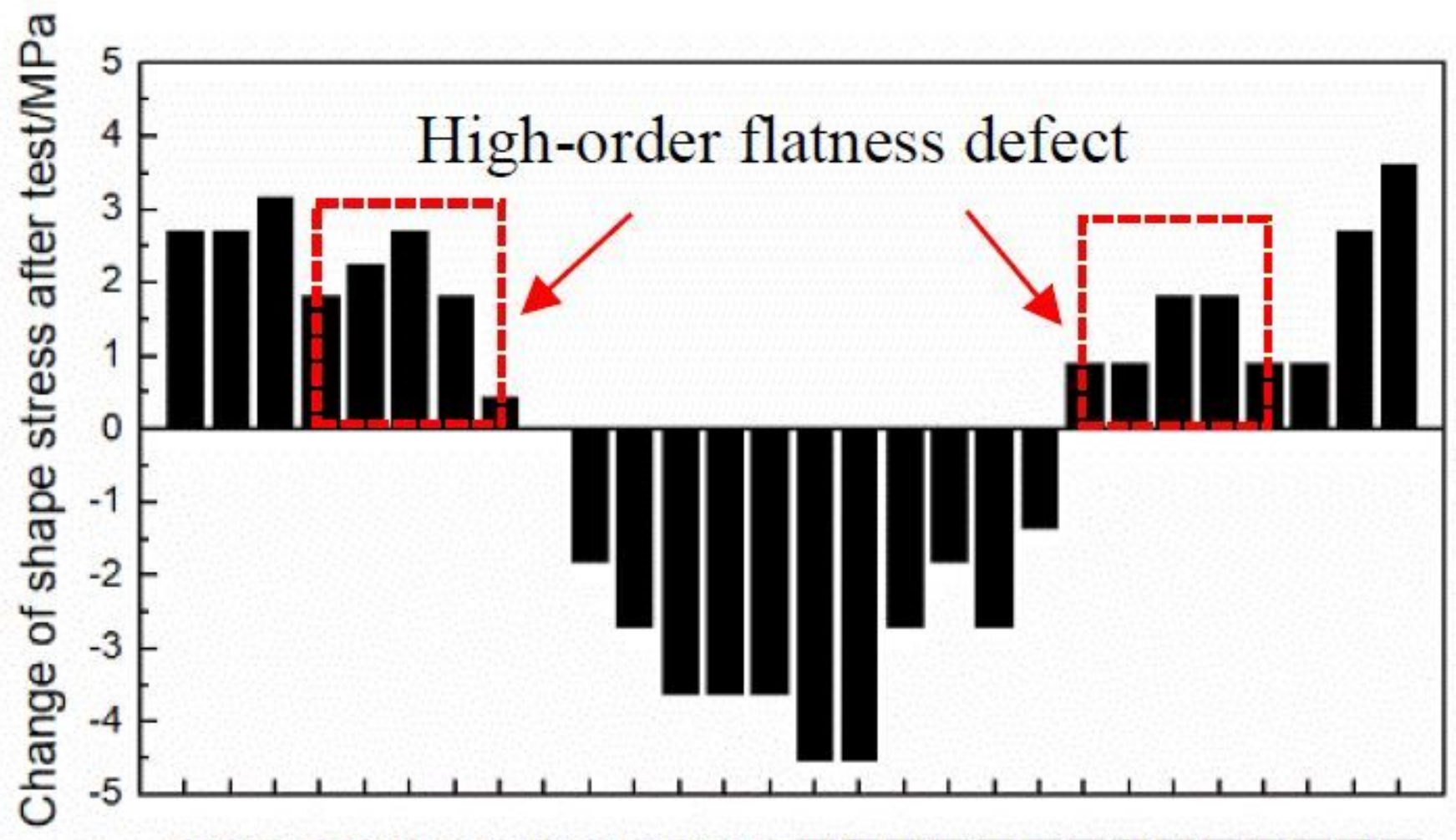

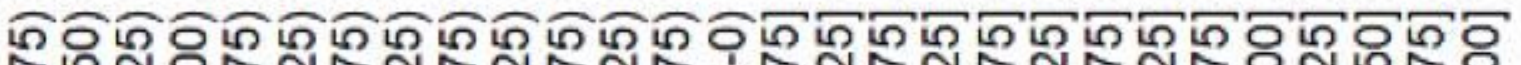
ถุ

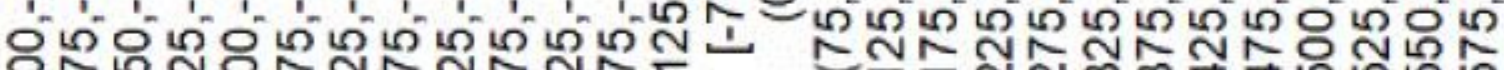

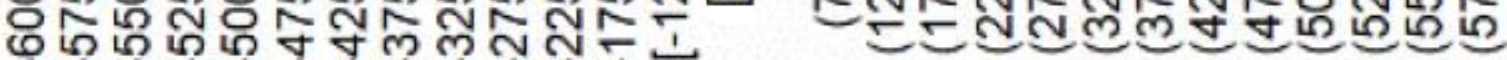
ำ

Measurement range of stress from the center of titanium strip/mm

Figure 15

The variation of stress distribution after industry test. 